\title{
Correções de Bartlett e Tipo-Bartlett em \\ Modelos Normais Heterocedásticos
}

\author{
Deusélio Bassini Fioresi
}

Dissertaçào apresentada ao Instituto de Matemática e Estatística

da Liniversidade de São Paulo para obtenção do grau de Mestre em Estatística

Área de concentração: Estatística

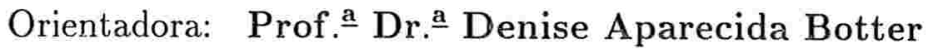

- São Paulo, janeiro de 2000 - 


\title{
Correções de Bartlett e Tipo-Bartlett em Modelos Normais Heterocedásticos
}

\author{
Este exemplar corresponde à redação final devidamen- \\ te corrigida e defendida por Deusélio Bassini Fioresi e \\ aprovada pela comissão julgadora.
}

São Paulo, 18 de janeiro de 2000.

Banca examinadora:

- Prof. ${ }^{\underline{a}}$ Dr. ${ }^{\underline{a}}$ Denise Aparecida Botter

- Prof. ${ }^{\mathrm{a}}$ Dr. ${ }^{\mathrm{a}}$ Lúcia Pereira Barroso

- Prof.. Dr.. ValdérioAnselmo Reisen. 
Aos meus pais, minha esposa e meus irmãos. 


\section{Agradecimentos}

Com a finalização deste trabalho, acrescento a meus conhecimentos uma experiência até então não vivenciada. Embora tenha encontrado algumas dificuldades neste primeiro trabalho de pesquisa, posso dizer que foi uma experiência bastante agradável, despertando interesse para novas pesquisas. A este feito. devo atribuir diversos fatores, incluindo o ambiente do IME-USP. Em especial, manifesto profunda gratidão

- à Professora Denise Aparecida Botter, a qual teve participação crucial como orientadora, além de sua dedicação e incentivo,

- ao meus pais e meus irmãos, que sempre contribuíram de várias formas para a continuidade de meus estudos e a minha noiva, agora esposa, que nunca deixou de me incentivar nos momentos difíceis,

- a todos os professores que deram alguma contribuição, direta ou indireta, para a realização deste trabalho,

- a todos os colegas de mestrado que, com o espírito de pesquisa, favoreceram meu crescimento acadêmico. Em particular, aos amigos Aldy Fernandes da Silva, por sua colaboração e incentivo e Demerval Soares Moreira, por seu auxílio inestimável na elaboração do programa de simulação,

- às agências CAPES, CNPq, FAPESP e FINEP, pelo apoio financeiro. 


\section{Sumário}

Resumo

$\begin{array}{lll}1 & \text { Introdução } & 7\end{array}$

$1.1 \mathrm{O}$ modelo normal heterocedástico . . . . . . . . . . . . . . . 9

1.2 Estimação dos parâmetros . . . . . . . . . . . . . . . . . 11

1.3 Hipóteses de interesse . . . . . . . . . . . . . . . . . . 13

2 Correção de Bartlett para a estatística da razão de verossimilhança

2.1 Introdução . . . . . . . . . . . . . . . . . . . . . . . . . 14

2.2 Testes simultâneos sobre a média e o parâmetro de precisão . . . . . . . . 17

2.2.1 Modelo de regressão linear simples . . . . . . . . . . . . . . . . 22

2.3 Testes sobre o parâmetro de precisão . . . . . . . . . . . . . . . . . 24

2.3 .1 Modelo de regressão linear simples . . . . . . . . . . . . . . 27 
2.4 Testes sobre a média . . . . . . . . . . . . . . . . .

3 Correção tipo-Bartlett para a estatística escore 33

3.1 Introdução . . . . . . . . . . . . . . . . . . . . 33

3.2 Testes simultâneos sobre a média e o parâmetro de precisão . . . . . . . . . . 34

3.2 .1 Modelo de regressào linear simples . . . . . . . . . . . . . . . . 40

3.3 Testes sobre o parâmetro de precisão . . . . . . . . . . . . . . . . 40

3.3.1 Modelo de regressào linear simples . . . . . . . . . . . . . . . . . 44

3.4 Testes sobre a média . . . . . . . . . . . . . . . . . . 45

4 Resultados de simulações e aplicação a dados reais 49

4.1 Resultados de simulações . . . . . . . . . . . . . . . . . . . . . . . 49

4.2 Uma aplicação a dados reais . . . . . . . . . . . . . . . . . . 57

$\begin{array}{ll}\text { Conclusão } & 59\end{array}$

$\begin{array}{ll}\text { Apêndice } & 60\end{array}$

Referências Bibliográficas $\quad 62$ 


\section{Resumo}

Neste trabalho, nos concentramos em encontrar fatores de correção de Bartlett para a estatística da razão de verossimilhança e tipo-Bartlett para a estatística escore, na classe dos modelos normais heterocedásticos com funções de ligação quaisquer para a média e para o parâmetro de precisão. Apresentamos. também, resultados de simulação e de uma aplicação a um conjunto de dados reais, ilustrando a utilidade dos fatores de correção obtidos em amostras finitas.

\section{Abstract}

In this work, we concentrate on finding Bartlett corrections for the likelihood ratio statistic and Bartlett-type corrections for the score statistic in the class of heteroscedastic normal models with any link functions for mean and precision parameters. We also present simulation results and an application to a set of real data illustrating the finite sample usefulness of the obtained corrections. 


\section{Capítulo 1}

\section{Introdução}

Da teoria sobre testes de hipóteses (ver, por exemplo, Cox e Hinkley, 1974, Seção 9.3) sabemos que, sob a hipótese nula, as distribuições das estatísticas da razão de verossimilhança $(L R)$ e escore $\left(S_{R}\right)$ podem ser aproximadas, para problemas regulares e em grandes amostras, pela distribuição qui-quadrado. Com relação à estatística da razão de verossimilhança. Bartlett (1937), Lawley (1956), Hayakawa (1977), Cordeiro (1987) e Chesher e Smith (1995). mostraram que é possível melhorar a qualidade da aproximação por qui-quadrado multiplicando-se a estatística $L R$ por um fator de correção, denominado fator de correção de Bartlett. Cordeiro e Ferrari (1991) derivaram uma fórmula geral para fatores de correção tipo-Bartlett que aperfeiçoam estatísticas cujas distribuições assintóticas são qui-quadrado centrais. O fator de correção de Bartlett que aperfeiģa a estatística $L R$ e o tipo-Bartlett que aperfeiçoa a estatística escore são casos particulares desta fórmula. As estatísticas aperfeiçoadas por fatores de correção de Bartlett ou tipo-Bartlett têm distribuições qui-quadrado com um erro de ordem $n^{-2}$, sob a hipótese nula, onde $n$ é o tamanho da amostra. Além disto, resultados de estudos de simulação como, por exemplo, os de Hollas (1991), Cordeiro (1993) e Cribari-Neto e Ferrari (1995a) mostraram que os testes de hipóteses baseados 
nas estatísticas $(L R)$ e $\left(S_{R}\right)$ modificadas têm tamanhos empíricos mais próximos dos tamanhos nominais do que os testes baseados nas estatísticas originais (não modificadas) quando amostras de tamanhos pequenos e moderados são consideradas.

Neste trabalho, apresentamos as estatísticas da razão de verossimilhança e escore, assim como suas versões aperfeiçoadas por fatores de correção de Bartlett e tipo-Bartlett, respectivamente, para vários testes de hipóteses em modelos normais heterocedásticos, considerando funções de ligação quaisquer para a média e para a variância. É bastante comum, na prática, que a média e a variância sejam modelados por diferentes funções de regressão. Para exemplificar. em experimentos de controle de qualidade na área industrial, é frequente procurar minimizar a variância enquanto se controla o valor da média do processo. Análises de dados provenientes de tais experimentos requerem, em geral, a modelagem conjunta da média e da variância. Para o modelo normal heterocedástico há vários trabalhos que tratam da obtenção de fatores de Bartlett e tipo-Bartlett para as estatísticas da razão de verossimilhança e escore, respectivamente. Dentre eles, destacamos os de Attfield (1991), Cordeiro (1993). Cribari-Neto e Ferrari (1995a,b), Botter e Cordeiro (1997) e Aubin e Cordeiro (1998). Cribari-Neto e Zarkos (1999) utilizaram métodos de simulação de Monte Carlo para estudarem o comportamento em amostras finitas de estimadores e testes do tipo "bootstrap"no modelo linear heterocedástico. Resultados de similação são apresentados para comparar o tamanho e o poder do teste escore de homocedasticidade de Breusch e Pagan (1979) com os correspondentes tamanhos e poderes dos testes escore corrigidos de Cribari-Neto e Ferrari (1995a) e Honda (1988).

No Capítulo 1, definimos o modelo normal heterocedástico, estabelecemos um método de estimação de parâmetros utilizando o processo iterativo escore de Fisher e definimos as hipóteses de interesse. No Capítulo 2, apresentamos correções de Bartlett para a estatística $L R$ e fazemos aplicações a casos especiais do modelo normal heterocedástico. Expressões para correções tipo-Bartlett para a estatística escore $S_{R}$ e aplicações a alguns casos especias, 
são apresentadas no Capítulo 3. Finalmente, no Capítulo 4, apresentamos resultados de simulação comparando os desempenhos das estatísticas $L R$ e $S_{R}$, em suas versões originais e aperfeiçoadas, respectivamente, por correções de Bartlett e tipo-Bartlett, para vários testes de hipóteses de interesse e fazemos uma aplicação a um conjunto de dados reais.

\subsection{O modelo normal heterocedástico}

Sejam $Y_{1}, \ldots, Y_{n}$ um conjunto de $n$ variáveis aleatórias independentes e normalmente distribuídas. Denotemos por $y_{l}$ o valor observado de $Y_{l}$ e por $y=\left(y_{1}, \ldots, y_{n}\right)^{T}$ o vetor das $n$ observações. A função densidade de probabilidade para $Y_{l}$ é dada por,

$$
f\left(y ; \mu_{l} ; \sigma_{l}^{2}\right)=\frac{1}{\sqrt{2 \pi \sigma_{l}^{2}}} \exp \left\{-\frac{1}{2 \sigma_{l}^{2}}\left(y_{l}-\mu_{l}\right)^{2}\right\},
$$

com $\mathrm{E}\left(Y_{l}\right)=\mu_{l}$ e $\operatorname{var}\left(Y_{l}\right)=\sigma_{l}{ }^{2}$, onde $\mu=\left(\mu_{1}, \ldots, \mu_{n}\right)^{T}$ está relacionado com o preditor linear $\eta=\left(\eta_{1}, \ldots, \eta_{n}\right)^{T}=X \beta$ através da função $d(\mu)=\eta$, sendo $X=\left(x_{1}, \ldots, x_{n}\right)^{T}$ uma matriz conhecida de dimensão $n \times p$ e posto completo $p(p<n)$, com $x_{l}=\left(x_{l 1}, \ldots, x_{l p}\right)^{T}, l=$ $1, \ldots, n$, e $\beta$ um vetor de dimensão $p \times 1$ de parâmetros de regressão desconhecidos. Definimos a componente sistemática para o parâmetro de precisão $\phi=\left(\phi_{1}, \ldots, \phi_{n}\right)^{T}$, com $\phi_{l}=1 / \sigma_{l}^{2}, l=1, \ldots, n$, como $g(\phi)=\tau=S \gamma$, sendo $S=\left(s_{1}, \ldots, s_{n}\right)^{T}$ uma matriz conhecida de dimensão $n \times q$ e posto completo $q(q<n)$ sendo $s_{l}=\left(s_{l 1}, \ldots, s_{l q}\right)^{T}$, e $\gamma$ um vetor de dimensão $q \times 1$ de parâmetros de regressão desconhecidos. Para fins de estimação e testes de hipóteses, segue que $Y=\left(y_{1}, \ldots, y_{n}\right)^{T}$ tem distribuição normal multivariada com média $\mu$ e matriz de variância-covariância $\Phi^{-1}=\operatorname{diag}\left\{\phi_{1}^{-1}, \ldots, \phi_{n}^{-1}\right\}$. As funções $d($.$) e g($.$) ,$ assumidas biunívocas, são denominadas funções de ligação para a média e para o parâmetro de precisão, respectivamente. Assumimos também que $\beta$ e $\gamma$ não têm elementos comuns. $O$ modelo descrito acima é denominado modelo normal heterocedástico.

O logaritmo da função de verossimilhança total, $\ell(\beta, \gamma)$, dado $y$, para a densidade em 
(1.1), pode ser expresso na forma aproximada

$$
\ell(\beta, \gamma) \cong-\frac{1}{2} \sum_{l=1}^{n}\left\{\phi_{l}\left(y_{l}-\mu_{l}\right)^{2}+\log \phi_{l}\right\} .
$$

A função escore total definida por

$$
U=U(\beta, \gamma)=\left(\begin{array}{c}
U_{\beta}(\beta, \gamma) \\
U_{\gamma}(\beta, \gamma)
\end{array}\right)=\left(\begin{array}{l}
\partial \ell(\beta, \gamma) / \partial \beta \\
\partial \ell(\beta, \gamma) / \partial \gamma
\end{array}\right)
$$

tem como componentes as primeiras derivadas da função $\ell(\beta, \gamma)$, em (1.2), em relação aos parâmetros $\beta$ e $\gamma$. Em notação matricial essas derivadas tomam as seguintes formas

$$
U_{\beta}(\beta, \gamma)=X^{T} \Phi W^{1 / 2}(y-\mu)
$$

e

$$
U_{\gamma}(\beta, \gamma)=-S^{T} \Phi_{1} \vartheta
$$

onde $W^{1 / 2}=\operatorname{diag}\left\{w_{1}^{1 / 2}, \ldots, w_{n}^{1 / 2}\right\}, \operatorname{com} w_{l}=\left(d \mu_{l} / d \eta_{l}\right)^{2}, \Phi=\operatorname{diag}\left\{\phi_{1}, \ldots, \phi_{n}\right\}, \Phi_{1}=$ $\operatorname{diag}\left\{\phi_{11}, \ldots, \phi_{1 n}\right\}, \operatorname{com} \phi_{1 l}=d \phi_{l} / d \tau_{l}$ e $\vartheta=\left(\vartheta_{1}, \ldots, \vartheta_{n}\right), \operatorname{com} \vartheta_{l}=1 / 2\left[\left(y_{l}-\mu_{l}\right)^{2}-1 / \phi_{l}\right], l=$ $1, \ldots, n$.

A matriz de informação total de Fisher $K=K(\beta, \gamma)$ para $\left(\beta^{T}, \gamma^{T}\right)^{T}$ é bloco-diagonal com submatrizes,

$$
K_{\beta, \beta}=-K_{\beta \beta}=E\left[-\frac{\partial^{2} \ell(\beta, \gamma)}{\partial \beta \partial \beta^{T}}\right]=X^{T} \Phi W X
$$

$\operatorname{para} \beta \mathrm{e}$

$$
K_{\gamma, \gamma}=-K_{\gamma \gamma}=E\left[-\frac{\partial^{2} \ell(\beta, \gamma)}{\partial \gamma \partial \gamma^{T}}\right]=\frac{1}{2} S^{T} \Phi^{-2} \Phi_{1}^{2} S
$$

para $\gamma$, onde $\Phi_{1}^{2}=\operatorname{diag}\left\{\phi_{11}^{2}, \ldots, \phi_{1 n}^{2}\right\}$ e $\Phi^{-2}=\operatorname{diag}\left\{\phi_{1}^{-2}, \ldots, \phi_{n}^{-2}\right\}$.

Como $K$ é bloco-diagonal, os parâmetros $\beta$ e $\gamma$ são globalmente ortogonais (ver, por exemplo, Cox e Reid, 1987) e seus estimadores de máxima verossimilhança $\hat{\beta}$ e $\hat{\gamma}$, respectivamente, são assintoticamente independentes. 


\subsection{Estimação dos parâmetros}

Sejam $K_{\beta, \beta}^{-1}$ e $K_{\gamma, \gamma}^{-1}$ as inversas das matrizes de informação total de Fisher para $\beta$ e $\gamma$, respectivamente. A inversa da matriz informação total de Fisher para $\left(\beta^{T}, \gamma^{T}\right)^{T}$ é dada por $K^{-1}=\operatorname{diag}\left\{K_{\beta, \beta}^{-1}, K_{\gamma, \gamma}^{-1}\right\}$.

Os estimadores de máxima verossimilhança $\hat{\beta}$ e $\hat{\gamma}$ podem ser calculados através do processo iterativo escore de Fisher

$$
\left[\begin{array}{l}
\hat{\beta}^{(m+1)} \\
\hat{\gamma}^{(m+1)}
\end{array}\right]=\left[\begin{array}{l}
\hat{\beta}^{(m)} \\
\hat{\gamma}^{(m)}
\end{array}\right]+\hat{K}^{(m)^{-1}} \hat{U}^{(m)},
$$

onde $\hat{K}^{(m)^{-1}}$ e $\hat{U}^{(m)^{-1}}$ são, respectivamente, a inversa da matriz de informação total de Fisher e a função escore avaliadas nas estimativas do passo $m$. Como $K^{-1}$ é bloco-diagonal, obtemos no passo $m$,

$$
\hat{\beta}^{(m+1)}=\hat{\beta}^{(m)}+\hat{K}_{\beta, \beta}^{(m)^{-1}} \hat{U}_{\beta}^{(m)}
$$

$\mathrm{e}$

$$
\hat{\gamma}^{(m+1)}=\hat{\gamma}^{(m)}+\hat{K}_{\gamma, \gamma}^{(m)^{-1}} \hat{U}_{\gamma}^{(m)} .
$$

Multiplicando ambos os lados de (1.7) por $\hat{K}_{\beta, \beta}^{(m)}$ temos

$$
\hat{K}_{\beta, \beta}^{(m)} \hat{\beta}^{(m+1)}=\hat{K}_{\beta, \beta}^{(m)} \hat{\beta}^{(m)}+\hat{U}_{\beta}^{(m)} .
$$

De (1.3) e (1.5), obtemos 


$$
\begin{aligned}
X^{T} \hat{\Phi}^{(m)} \hat{W}^{(m)} X \hat{\beta}^{(m+1)} & =X^{T} \hat{\Phi}^{(m)} \hat{W}^{(m)} X \hat{\beta}^{(m)}+X^{T} \hat{\Phi}^{(m)} \hat{W}^{(m)^{1 / 2}}(y-\hat{\mu})^{(m)} \\
& =X^{T} \hat{\Phi}^{(m)} \hat{W}^{(m)}\left[X \hat{\beta}^{(m)}+\hat{W}^{(m)^{-1 / 2}}(y-\hat{\mu})^{(m)}\right] .
\end{aligned}
$$

Definindo $\quad \hat{z}^{(m)}=X \hat{\beta}^{(m)}+\hat{W}^{(m)^{-1 / 2}}(y-\hat{\mu})^{(m)}$, escrevemos (1.9) como

$$
\hat{\beta}^{(m+1)}=\left(X^{T} \hat{\Phi}^{(m)} \hat{W}^{(m)} X\right)^{-1} X^{T} \hat{\Phi}^{(m)} \hat{W}^{(m)} \hat{z}^{(m)}
$$

Através de um desenvolvimento análogo ao descrito acima para a obtenção de $\hat{\beta}^{(m+1)}$, encontramos a partir de (1.4), (1.6) e (1.8)

$$
\hat{\gamma}^{(m+1)}=\left(S^{T} \hat{\Phi}^{-2(m)} \hat{\Phi}_{1}^{2(m)} S\right)^{-1} S^{T} \hat{\Phi}^{-2(m)} \hat{\Phi}_{1}^{2(m)} \hat{r}^{(m)},
$$

onde

$$
\hat{r}^{(m)}=S \hat{\gamma}^{(m)}+2 \hat{\Phi}^{2(m)} \hat{\Phi}_{1}^{-1(m)} \hat{\vartheta}^{(m)}
$$

As expressões (1.10) e (1.11) são simplesmente os vetores de coeficientes da regressão de mínimos quadrados ponderados de $z$ sobre as colunas de $X$ com $\Phi W$ como matriz de peso para $\beta$, e de $r$ sobre as colunas de $S \operatorname{com} \Phi^{-2} \Phi_{1}^{2}$ como matriz de peso para $\gamma$.

Inicializando o processo com estimativas $\hat{\beta}^{(0)}$ e $\hat{\gamma}^{(0)}$ obtemos, através de (1.10) e (1.11), os estimadores de máxima verossimilhança para $\beta$ e $\gamma$ no passo $m=1$, que reiniciarão o processo para a obtenção das estimativas do passo $m=2$ e assim, sucessivamente, até que a convergência seja obtida. Smyth (1989), de acordo com Nelder e Wedderburn (1972), sugere que $\hat{\beta}^{(0)}$ seja obtido assumindo em $(1.10) \mu_{l}=y_{l}$ e $\phi_{l}=1$, enquanto que $\hat{\gamma}^{(0)}$ seja obtido assumindo em (1.11) $\phi_{l}=\left(y_{l}-\hat{\mu}_{l}\right)^{2}$. 


\subsection{Hipóteses de interesse}

Consideremos as partições $\beta=\left(\beta_{1}{ }^{T}, \beta_{2}{ }^{T}\right)^{T}$ e $\gamma=\left(\gamma_{1}{ }^{T}, \gamma_{2}{ }^{T}\right)^{T}$, onde $\beta_{1}=\left(\beta_{1}, \ldots, \beta_{p_{1}}\right)^{T}$, $\beta_{2}=\left(\beta_{p_{1}+1}, \ldots, \beta_{p}\right)^{T}, \gamma_{1}=\left(\gamma_{1}, \ldots, \gamma_{q_{1}}\right)^{T}$ e $\gamma_{2}=\left(\gamma_{q_{1}+1}, \ldots, \gamma_{q}\right)^{T}$ com $p_{1} \leq p$ e $q_{1} \leq q$. Para o modelo em (1.1), estamos interessados no teste da hipótese $H_{1}: \beta_{1}=\beta_{1}{ }^{(0)}, \gamma_{1}=\gamma_{1}{ }^{(0)}$ contra $H$ : pelo menos uma das igualdades é violada. onde $\beta_{1}{ }^{(0)}$ e ${\gamma_{1}}^{(0)}$ são vetores especificados de dimensões $p_{1}$ e $q_{1}$, respectivamente. Considerando $p_{1}=p-1$ e $q_{1}=q-1$, queremos testar, em particular, a hipótese $H^{\prime}{ }_{1}: \beta_{1}=0, \gamma_{1}=0, \operatorname{com} x_{l p}=1$ e $s_{l q}=1$, para $l=1, \ldots, n$. O caso $p_{1}=0$ torna possível o teste de hipótese $H_{2}: \gamma_{1}=\gamma_{1}{ }^{(0)}$. Supondo $p_{1}=0$ e $q_{1}=q-1$, um caso especial refere-se ao teste de ${H^{\prime}}_{2}: \gamma_{1}=0$, com $s_{l q}=1$, para $l=1, \ldots, n$. Para $q_{1}=0$, temos interesse no teste de $H_{3}: \beta_{1}=\beta_{1}{ }^{(0)}$. Supondo $p_{1}=p-1$ e $q_{1}=0$, temos como caso especial o teste de $H^{\prime}{ }_{3}: \beta_{1}=0$, com $x_{l p}=1$, para $l=1, \ldots, n$. Observemos que as hipóteses $H_{2}, H_{3}, H^{\prime}{ }_{1}, H^{\prime}{ }_{2}$ e $H^{\prime}{ }_{3}$ devem ser testadas contra a hipótese alternativa $H$ definida anteriormente. $\mathrm{O}$ teste de $H^{\prime}{ }_{1}$ equivale a testar se as variáveis aleatórias $Y_{1}, \ldots, Y_{n}$ são identicamente distribuídas. Já o teste de ${H^{\prime}}_{2}$ se reduz ao teste de homocedasticidade, ou seja, equivale a testar se $Y_{1}, \ldots, Y_{n}$ têm a mesma variância. Este teste tem sido muito explorado na literatura (ver, por exemplo, Godfrey, 1978; Breusch e Pagan, 1979; Cook e Weisberg, 1983, Attfield, 1991 e Jeffrey e Chih-Ling 1994). Finalmente, o teste de $H^{\prime}{ }_{3}$ equivale a verificar se $Y_{1}, \ldots, Y_{n}$ têm a mesma média, isto é, se $\mu_{l}=d^{-1}\left(\beta_{p}\right)$, para $l=1, \ldots, n$. 


\section{Capítulo 2}

\section{Correção de Bartlett para a estatística da razão de verossimilhança}

\subsection{Introdução}

A estatística da razão de verossimilhança $L R$ é freqüentemente utilizada em testes de hipóteses. Sabemos, que sob a hipótese nula, $L R$ tem distribuição aproximadamente $\chi^{2}$ com $\psi$ graus de liberdade, onde $\psi$ é a diferença entre as dimensões dos espaços paramétricos associados as hipóteses testadas (ver, por exemplo, Cox e Hinkley, 1974, Seção 9.3). De acordo com Bartlett (1937), essa aproximação para a distribuição $\chi^{2}$ pode ser melhorada substituindo $L R$ pela estatística $L R^{*}=c^{-1} L R$, onde $c$ é uma constante, denominada correção de Bartlett, determinada pela relação $c=\psi^{-1} E(L R)$, onde $E(L R)$, o valor esperado da estatística $L R$, é calculado com um erro de ordem $O\left(n^{-2}\right)$, sendo $n$ o tamanho da amostra. É importante salientar que, quando afirmamos que a distribuição de $L R^{*}$ é $\chi_{\psi}^{2}$ até ordem $n^{-1}$ (o erro da aproximação é de ordem $O\left(n^{-2}\right)$ ), sob a hipótese nula, estamos supondo 
que certas condições de regularidade estão satisfeitas. Uma discussão detalhada destas suposições pode ser encontrada em Chandra e Ghosh (1979), Chandra (1985) e Jensen (1993). No caso da correção $c$ depender de parâmetros desconhecidos, estes devem ser substituídos por suas respectivas estimativas de máxima verossimilhança sob a hipótese nula. Neste caso, a estatística corrigida resultante ainda tem distribuição $\chi_{\psi}^{2}$ até ordem $n^{-1}$ sob a hipótese nula (Lawley, 1956).

O objetivo deste capítulo é obter correções de Bartlett, em forma matricial, para as estatísticas da razão de verossimilhança associadas aos testes das hipóteses $H_{1}, H_{2}$ e $H_{3}$ descritas na Seção 1.2. Baseados no trabalho de Lawley (1956), dispomos de uma fórmula matricial para o valor esperado $2 E\{\ell(\hat{\beta}, \hat{\gamma})-\ell(\beta, \gamma)\}$ até ordem $O\left(n^{-1}\right)$, onde $\ell(\beta, \gamma)$ é o logaritmo da função de verossimilhança dado em (1.2) e $\hat{\beta}$ e $\hat{\gamma}$ são os estimadores de máxima verossimilhança de $\beta$ e $\gamma$, respectivamente, sob o modelo irrestrito. Nas Seções 2.2 a 2.4 , esta fórmula será utilizada para discutir os testes da razão de verossimilhança das hipóteses $H_{1}, H_{2}$ e $H_{3}$ aperfeiçoados por correções de Bartlett.

De acordo com Lawley(1956), podemos escrever até ordem $O\left(n^{-1}\right)$

$$
E\{2[\ell(\hat{\beta}, \hat{\gamma})-\ell(\beta, \gamma)]\}=p+q+\varepsilon_{p+q}
$$

onde o termo $\varepsilon_{p+q}$ é de ordem $O\left(n^{-1}\right)$ e $p$ e $q$ são as dimensões dos vetores $\beta$ e $\gamma$, respectivamente. O termo $\varepsilon_{p+q}$ é obtido através de cumulantes conjuntos de derivadas de $\ell(\beta, \gamma)$ com respeito a componentes de $\beta$ e $\gamma$.

Botter e Cordeiro (1997) apresentam uma fórmula para o termo $\varepsilon_{p+q}$ em modelos lineares generalizados com componente sistemática para o parâmetro de dispersão. De acordo com esse resultado. a fórmula de $\varepsilon_{p+q}$ para o modelo normal heterocedástico em (1.1) é dada por

$$
\varepsilon_{p+q}=\varepsilon_{\beta p}+\varepsilon_{\gamma q}+\frac{1}{2} \operatorname{tr}\left(\Phi_{2} W Z_{\beta d} Z_{\gamma d}-\Phi_{1} W Z_{\beta}^{(2)} \Phi_{1} W Z_{\gamma}\right)
$$




$$
+\frac{1}{4} 1^{T} \Phi_{1} W Z_{\beta d} Z_{\gamma}\left\{Z_{\beta d} W+Z_{\gamma d}\left(2 \Phi^{3} \Phi_{1}^{2}-\Phi^{2} \Phi_{2}\right)\right\} \Phi_{1} 1
$$

com

$$
\varepsilon_{\beta p}=\frac{1}{4} \operatorname{tr}\left(\Phi^{-1} H Z_{\beta d}^{2}\right)+\frac{1}{4} 1^{T} \Phi^{-1} F\left(-2 Z_{\beta}^{(3)}+Z_{\beta d} Z_{\beta} Z_{\beta d}\right) F \Phi^{-1} 1
$$

e

$$
\begin{aligned}
\varepsilon_{\gamma q}= & \frac{1}{4} \operatorname{tr}\left(\Phi_{3} Z_{\gamma d}^{2}\right)+\frac{1}{12} 1^{T} \Phi^{3} \Phi_{1}^{3}\left(2 Z_{\gamma}^{(3)}+3 Z_{\gamma d} Z_{\gamma} Z_{\gamma d}\right) \Phi_{1}^{3} \Phi^{3} 1 \\
& +\frac{1}{16} 1^{T} \Phi^{2} \Phi_{1} \Phi_{2}\left(Z_{\gamma d} Z_{\gamma} Z_{\gamma d}-2 Z_{\gamma}^{(3)}\right) \Phi_{2} \Phi_{1} \Phi^{2} 1 \\
& -\frac{1}{4} 1^{T} \Phi^{3} \Phi_{1}^{3} Z_{\gamma d} Z_{\gamma} Z_{\gamma d} \Phi_{1} \Phi_{2} \Phi^{2} 1
\end{aligned}
$$

onde $\Phi=\operatorname{diag}\left\{\phi_{1}, \ldots, \phi_{n}\right\}, \Phi_{1}=\operatorname{diag}\left\{\phi_{11}, \ldots, \phi_{1 n}\right\}, \Phi_{2}=\operatorname{diag}\left\{\phi_{21}, \ldots, \phi_{2 n}\right\}$, com $\phi_{1 l}=$ $d \phi_{l} / d \tau_{l}$ e $\phi_{2 l}=d^{2} \phi_{l} / d \tau_{l}^{2}, Z_{\beta}=\left(z_{\beta l m}\right)=X K_{\beta, \beta}^{-1} X^{T}$, para $K_{\beta, \beta}^{-1}$ definida no Capítulo 1, $Z_{\beta d}=\operatorname{diag}\left\{z_{\beta_{11}}, \ldots, z_{\beta_{n n}}\right\}, Z_{\beta d}^{2}=\operatorname{diag}\left\{z_{\beta_{11}}^{2}, \ldots, z_{\beta_{n n}}^{2}\right\}, Z_{\beta}^{2}=\left(z_{\beta l m}^{2}\right), Z_{\gamma}=\left(z_{\gamma l m}\right)=S K_{\gamma, \gamma}^{-1} S^{T}$, para $K_{\gamma, \gamma}^{-1}$ definida no Capítulo $1, Z_{\gamma d}=\operatorname{diag}\left\{z_{\gamma_{11}}, \ldots, z_{\gamma_{n n}}\right\}, Z_{\gamma d}^{2}=\operatorname{diag}\left\{z_{\gamma_{11}}^{2}, \ldots, z_{\gamma_{n n}}^{2}\right\}$, $\Phi_{1}^{3}=\operatorname{diag}\left\{\phi_{11}^{3}, \ldots, \phi_{1 n}^{3}\right\}, Z_{\beta}^{(3)}=\left(z_{\beta l m}^{3}\right), Z_{\gamma}^{(3)}=\left(z_{\gamma l m}^{3}\right), 1$ é um vetor $n \times 1$ de valores iguais a 1 e $\Phi_{3}, F$ e $H$, são matrizes diagonais $n \times n$, dadas por $\Phi_{3}=\operatorname{diag}\left\{\phi_{31}, \cdots, \phi_{3 n}\right\}$, para $\phi_{3 l}=$ $\left(3 / \phi_{l}^{4}\right)\left(d \phi_{l} / d \tau_{l}\right)^{4}-2\left(1 / \phi_{l}^{3}\right)\left(d \phi_{l} / d \tau_{l}\right)^{2}\left(d^{2} \phi_{l} / d \tau_{l}^{2}\right)-\left[1 /\left(2 \phi_{l}^{2}\right)\right]\left(d^{2} \phi_{l} / d \tau_{l}^{2}\right)^{2}, F=\operatorname{diag}\left\{f_{1}, \cdots, f_{n}\right\}$, $\operatorname{com} f_{l}=\left(d \mu_{l} / d \eta_{l}\right)\left(d^{2} \mu_{l} / d \eta_{l}^{2}\right)$, e $H=\operatorname{diag}\left\{h_{1}, \cdots, h_{n}\right\}$, para $h_{l}=\left(d^{2} \mu_{l} / d \eta_{l}^{2}\right)^{2}$. As matrizes $Z_{\beta}$ e $Z_{\gamma}$ são, respectivamente, as matrizes de covariância assintóticas de $\hat{\eta}=X \hat{\beta}$ e $\hat{\tau}=S \hat{\gamma}$.

Considerando um modelo com função de ligação identidade para a média, $\mu=\eta=X \beta$, e função de ligação logaritmica para o parâmetro de precisão, $-\log \phi=\tau=S \gamma$, temos $w_{l}=1, f_{l}=h_{l}=0, d\left(\phi_{l}\right)=(-1 / 2) \log \phi_{l},-\phi_{1 l}=\phi_{2 l}=\phi_{l}$ e $\phi_{3 l}=-1 / 2$, para $l, \ldots, n$. Neste caso a expressão (2.3) se anula e as expressões (2.2) e (2.4) têm as seguintes formas, respectivamente,

$$
\varepsilon_{p+q}=\varepsilon_{\gamma q}+\frac{1}{2} \operatorname{tr}\left(\Phi^{-1} Z_{\beta d} Z_{\gamma d}-\Phi^{-1} Z_{\beta}^{(2)} \Phi^{-1} Z_{\gamma}\right)
$$




$$
+\frac{1}{4} 1^{T} \Phi^{-1} Z_{\beta d} Z_{\gamma} Z_{\beta d} \Phi^{-1} 1+\frac{1}{4} 1^{T} \Phi^{-1} Z_{\beta d} Z_{\gamma} Z_{\gamma d} 1
$$

$\mathrm{e}$

$$
\varepsilon_{\gamma q}=-\frac{1}{8} \operatorname{tr}\left(Z_{\gamma d}^{2}\right)+\frac{1}{24} 1^{T} Z_{\gamma}^{(3)} 1+\frac{1}{16} 1^{T} Z_{\gamma d} Z_{\gamma} Z_{\gamma d} 1
$$

Notemos que a expressão $\varepsilon_{\beta p}$, em (2.3) depende das matrizes $X$ e $S$ e dos parâmetros $\beta$ e $\gamma$, enquanto que a expressão $\varepsilon_{\gamma q}$ em (2.4) não envolve a matriz modelo $X$ nem o vetor $\beta$, dependendo somente da matriz $S$, do parâmetro $\gamma$ e de derivadas do parâmetro $\phi$ em relação à função de ligação $\tau$.

Para os modelos normais heterocedásticos que possuem matrizes de covariância assintóticas $Z_{\beta}$ e $Z_{\gamma}$ em forma fechada, podemos obter expressões de $\varepsilon_{p+q}$ em forma fechada. Nestas situações, os fatores de correção de Barttlet associados às estatísticas da razão de verossimilhança de vários testes de hipóteses também têm forma fechada. Além disso, as quantidades (2.2) - (2.4) podem ser facilmente obtidas através dos pacotes computacionais MATHEMATICA (Wolfram, 1991) ou MAPLE (Char et al.,1988).

\subsection{Testes simultâneos sobre a média e o parâmetro de precisão}

Nesta seção obtemos correções de Bartlett para estatísticas da razão de verossimilhança em testes de hipóteses simultâneos sobre a média e o parâmetro de precisão, ou seja, sobre $\left(\beta^{T}, \gamma^{T}\right)^{T}$, nos modelos normais heterocedásticos definidos em (1.1).

Consideremos as funções de ligação $d(\mu)$ e $g(\phi)$ definidas na Seção 1.2 e as partições $\beta^{T}=$ $\left(\beta_{1}{ }^{T}, \beta_{2}{ }^{T}\right)$ para o vetor $\beta$ e $\gamma^{T}=\left(\gamma_{1}{ }^{T}, \gamma_{2}{ }^{T}\right)$ para o vetor $\gamma$, onde $\beta_{1}=\left(\beta_{1}, \ldots, \beta_{p 1}\right)^{T}, \beta_{2}=$ 
$\left(\beta_{p 1+1}, \ldots, \beta_{p}\right)^{T}, \gamma_{1}=\left(\gamma_{1}, \ldots, \gamma_{q 1}\right)^{T}$ e $\gamma_{2}=\left(\gamma_{q 1+1}, \ldots, \gamma_{q}\right)^{T}$, com $p_{1} \leq p$ e $q_{1} \leq q$. Essas decomposições induzem as correspondentes partições

$$
\begin{aligned}
& X=\left(X_{1}, X_{2}\right), S=\left(S_{1}, S_{2}\right), \\
& U=\left(U_{\beta_{1}}^{T}\left(\beta_{1}, \beta_{2}, \gamma_{1}, \gamma_{2}\right), U_{\beta_{2}}^{T}\left(\beta_{1}, \beta_{2}, \gamma_{1}, \gamma_{2}\right), U_{\gamma_{1}}^{T}\left(\beta_{1}, \beta_{2}, \gamma_{1}, \gamma_{2}\right), U_{\gamma_{2}}^{T}\left(\beta_{1}, \beta_{2}, \gamma_{1}, \gamma_{2}\right)\right)^{T}, \\
& K_{\beta, \beta}=\left(\begin{array}{ll}
K_{\beta_{11}} & K_{\beta_{12}} \\
K_{\beta_{21}} & K_{\beta_{22}}
\end{array}\right) \quad \text { e } \quad K_{\gamma, \gamma}=\left(\begin{array}{cc}
K_{\gamma_{11}} & K_{\gamma_{12}} \\
K_{\gamma_{21}} & K_{\gamma_{22}}
\end{array}\right) \text {, }
\end{aligned}
$$

onde $X_{1}, X_{2}, S_{1}$ e $S_{2}$ são matrizes conhecidas de posto completo e dimensões $n \times p_{1}, n \times(p-$ $\left.p_{1}\right), n \times q_{1}, n \times\left(q-q_{1}\right)$, respectivamente, $K_{\beta_{11}}=X_{1}^{T} W \Phi X_{1}, K_{\beta_{12}}=K_{\beta_{21}}^{T}=X_{1}^{T} W \Phi X_{2}, K_{\beta_{22}}=$ $X_{2}^{T} W \Phi X_{2}, K_{\gamma_{11}}=2^{-1} S^{T}{ }_{1} \Phi^{-2} \Phi_{1}^{2} S_{1}, K_{\gamma_{12}}=K_{\gamma_{21}}^{T}=2^{-1} S_{1}^{T} \Phi^{-2} \Phi_{1}^{2} S_{2}$ e $K_{\gamma_{22}}=2^{-1} S_{2}^{T} \Phi^{-2} \Phi_{1}^{2} S_{2}$.

Estamos interessados em testar a hipótese $H_{1}: \beta_{1}=\beta_{1}{ }^{(0)}, \gamma_{1}=\gamma_{1}{ }^{(0)}$ contra $H$ : pelo menos uma das igualdades é violada, onde $\beta_{1}{ }^{(0)}$ e $\gamma_{1}{ }^{(0)}$ são vetores conhecidos de dimensões $p_{1}$ e $q_{1}$, respectivamente. Denotamos os estimadores de máxima verossimilhança dos parâmetros $\beta_{2}, \gamma_{2}$, sob $H_{1}$, com um acento ${ }^{\sim}$. A estatística da razão de verossimilhança para o teste de $H_{1}$ é dada por

$$
L R_{1}=2\left\{\ell\left(\hat{\beta}_{1}, \hat{\beta}_{2}, \hat{\gamma}_{1}, \hat{\gamma}_{2}\right)-\ell\left(\beta_{1}^{(0)}, \tilde{\beta}_{2}, \gamma_{1}^{(0)}, \tilde{\gamma}_{2}\right)\right\}
$$

que, sob $H_{1}$, tem distribuição assintótica $\chi_{p_{1}+q_{1}}^{2}$.

Para obtermos a correção de Bartlett para $L R_{1}$, escrevemos

$$
\begin{aligned}
L R_{1}= & 2\left[\left\{\ell\left(\hat{\beta}_{1}, \hat{\beta}_{2}, \hat{\gamma}_{1}, \hat{\gamma}_{2}\right)-\ell\left(\beta_{1}, \beta_{2}, \gamma_{1}, \gamma_{2}\right)\right\}\right. \\
& \left.-\left\{\ell\left(\beta_{1}^{(0)}, \tilde{\beta}_{2}, \gamma_{1}^{(0)}, \tilde{\gamma}_{2}\right)-\ell\left(\beta_{1}, \beta_{2}, \gamma_{1}, \gamma_{2}\right)\right\}\right],
\end{aligned}
$$

onde $\ell\left(\beta_{1}, \beta_{2}, \gamma_{1}, \gamma_{2}\right)$ é o logaritmo da função de verossimilhança obtida nos parâmetros $\beta$ e $\gamma$. 
A partir de (2.1) a (2.4) e de Botter e Cordeiro (1997) encontramos, até ordem $O\left(n^{-1}\right)$,

$$
2 E\left\{\ell\left(\hat{\beta}_{1}, \hat{\beta}_{2}, \hat{\gamma}_{1}, \hat{\gamma}_{2}\right)-\ell\left(\beta_{1}^{(0)}, \beta_{2}, \gamma_{1}^{(0)}, \gamma_{2}\right)\right\}=p+q+\varepsilon_{p+q}
$$

e

$$
2 E\left\{\ell\left(\beta_{1}^{(0)}, \tilde{\beta}_{2}, \gamma_{1}^{(0)}, \tilde{\gamma}_{2}\right)-\ell\left(\beta_{1}^{(0)}, \beta_{2}, \gamma_{1}^{(0)}, \gamma_{2}\right)\right\}=\left(p-p_{1}\right)+\left(q-q_{1}\right)+\varepsilon_{\left(p-p_{1}\right)+\left(q-q_{1}\right)},
$$

ou seja,

$$
\mathrm{E}\left(L R_{1}\right)=p_{1}+q_{1}+\varepsilon_{p+q}-\varepsilon_{(p+q)-\left(p_{1}+q_{1}\right)}
$$

onde

$$
\begin{aligned}
\varepsilon_{p+q}-\varepsilon_{(p+q)-\left(p_{1}+q_{1}\right)}= & \varepsilon_{3_{p}}-\varepsilon_{\beta\left(p-p_{1}\right)}+\varepsilon_{\gamma_{q}}-\varepsilon_{\gamma\left(q-q_{1}\right)}+\frac{1}{2} \operatorname{tr}\left\{\Phi _ { 2 } W \left(Z_{\beta d} Z_{\gamma d}\right.\right. \\
& \left.\left.-Z_{2 \beta d} Z_{2 \gamma d}\right)-\Phi_{1} W\left(Z_{\beta}^{(2)} \Phi_{1} W Z_{\gamma}-Z_{2 \beta}^{(2)} \Phi_{1} W Z_{2 \gamma}\right)\right\} \\
& +\frac{1}{4} 1^{T} \Phi_{1} W\left(Z_{\beta d} Z_{\gamma} Z_{\beta d}-Z_{2 \beta d} Z_{2 \gamma} Z_{2 \beta d}\right) W \Phi_{1} 1+\frac{1}{4} 1^{T} \Phi_{1} W \\
& \left(Z_{\beta d} Z_{\gamma} Z_{\gamma d}-Z_{2 \beta d} Z_{2 \gamma} Z_{2 \gamma d}\right)\left(2 \Phi^{-3} \Phi_{1}^{2}-\Phi^{-2} \Phi_{2}\right) \Phi_{1} 1,
\end{aligned}
$$

com

$$
\begin{aligned}
\varepsilon_{\beta_{p}}-\varepsilon_{\beta\left(p-p_{1}\right)}= & \frac{1}{4} \operatorname{tr}\left\{\Phi^{-1} H\left(Z_{\beta d}^{2}-Z_{2 \beta d}^{2}\right)\right\}+\frac{1}{4} 1^{T} \Phi^{-1} F\left\{-2\left(Z_{\beta}^{(3)}\right.\right. \\
& \left.\left.-Z_{2 \beta}^{(3)}\right)+\left(Z_{\beta d} Z_{\beta} Z_{\beta d}-Z_{2 \beta d} Z_{2 \beta} Z_{2 \beta d}\right)\right\} F \Phi^{-1} 1
\end{aligned}
$$

e

$$
\begin{aligned}
\varepsilon_{\gamma_{q}}-\varepsilon_{\gamma\left(q-q_{1}\right)}= & \frac{1}{4} \operatorname{tr}\left\{\Phi_{3}\left(Z_{\gamma, d}^{2}-Z_{2 \gamma d}^{2}\right)\right\}+\frac{1}{24} 1^{T} \Phi^{-3} \Phi_{1}^{3}\left\{4\left(Z_{\gamma}^{(3)}-Z_{2 \gamma}^{(3)}\right)+6\left(Z_{\gamma d} Z_{\gamma} Z_{\gamma d}\right.\right. \\
& \left.\left.-Z_{2 \gamma d} Z_{2 \gamma} Z_{2 \gamma d}\right)\right\} \Phi_{1}^{3} \Phi^{-3} 1+\frac{1}{16} 1^{T} \Phi^{-2} \Phi_{1} \Phi_{2}\left\{\left(Z_{\gamma d} Z_{\gamma} Z_{\gamma d}-Z_{2 \gamma d} Z_{2 \gamma} Z_{2 \gamma d}\right)\right.
\end{aligned}
$$




$$
\begin{aligned}
& \left.\left.-Z_{2 \gamma d} Z_{2 \gamma} Z_{2 \gamma d}\right)-2\left(Z_{\gamma}^{(3)}-Z_{2 \gamma}^{(3)}\right)\right\} \Phi_{2} \Phi_{1} \Phi^{-2} 1 \\
& -\frac{1}{4} 1^{T} \Phi^{-3} \Phi_{1}^{3}\left(Z_{\gamma d} Z_{\gamma} Z_{\gamma d}-Z_{2 \gamma d} Z_{2 \gamma} Z_{2 \gamma d}\right) \Phi_{1} \Phi_{2} \Phi^{-2} 1,
\end{aligned}
$$

sendo $Z_{2 \beta}=X_{2} K_{\beta_{22}}^{-1} X_{2}^{T}=X_{2}\left(X_{2}^{T} W \Phi^{-1} X_{2}\right)^{-1} X_{2}^{T}$ a matriz de covariância assintótica de $\tilde{\eta}=X_{1} \beta_{1}^{(0)}+X_{2} \tilde{\beta}_{2}, Z_{2 \beta d}=\operatorname{diag}\left\{z_{2 \beta_{11}}, \ldots, z_{2 \beta_{n n}}\right\}, Z_{2 \beta d}^{2}=\operatorname{diag}\left\{z_{2 \beta_{11}}^{2}, \ldots, z_{2 \beta_{n n}}^{2}\right\}, Z_{2 \beta}^{(2)}=$ $\left(z_{2 \beta_{l m}}^{2}\right), Z_{2 \beta}^{(3)}=\left(z_{2 \beta_{l m}}^{3}\right), Z_{2 \gamma}=S_{2} K_{\gamma_{22}}^{-1} S_{2}^{T}=2^{-1} S_{2}\left(S_{2}^{T} \Phi^{-2} \Phi_{1}^{2} S_{2}\right)^{-1} S_{2}^{T}$ a matriz de covariância assintótica de $\tilde{\tau}=S_{1} \gamma_{1}^{(0)}+S_{2} \tilde{\gamma}_{2}, Z_{2 \gamma d}=\operatorname{diag}\left\{z_{2_{\gamma 11}}, \ldots, z_{2_{\gamma n n}}\right\}, Z_{2 \gamma d}^{2}=\operatorname{diag}\left\{z_{2_{\gamma 11}}^{2}, \ldots, z_{2_{\gamma n n}}^{2}\right\}$ e $Z_{2 \gamma}^{(3)}=\left(z_{2 \gamma_{l m}}^{3}\right)$. Todas as matrizes diagonais $W, F, H, \Phi, \Phi_{1}, \Phi_{2}$ e $\Phi_{3}$ devem ser avaliadas sob o modelo restrito especificado por $H_{1}$.

A correção de Barttlet $c$ associada à estatística $L R_{1}$ é determinada pela equação

$$
c=\frac{E\left(L R_{1}\right)}{p_{1}+q_{1}}
$$

com $E\left(L R_{1}\right)$ dada por (2.7), ou seja,

$$
c=1+\frac{\varepsilon_{p+q}-\varepsilon_{(p+q)-\left(p_{1}+q_{1}\right)}}{p_{1}+q_{1}} .
$$

Assim a estatística corrigida $L R_{1}^{*}=\tilde{c}^{-1} L R_{1}$ tem distribuição $\chi_{p_{1}+q_{1}}^{2}$ até ordem $O\left(n^{-1}\right)$, sob $H_{1}$.

Como uma aplicação do resultado (2.11), consideremos o teste da hipótese nula $H^{\prime}{ }_{1}: \beta_{1}=$ $0, \gamma_{1}=0, \operatorname{com} p_{1}=p-1, q_{1}=q-1, x_{l p}=1$ e $s_{l q}=1$ para $l=1, \ldots, n$ contra a hipótese alternativa $H$, definida no início desta seção. A estatística da razão de verossimilhança associada a este teste é dada por

$$
L R_{1}^{\prime}=2\left\{\ell\left(\hat{\beta}_{1}, \hat{\beta}_{2}, \hat{\gamma}_{1}, \hat{\gamma}_{2}\right)-\ell\left(0, \tilde{\beta}_{p}, 0, \tilde{\gamma}_{q}\right)\right\}
$$


que, assintoticamente, tem distribuição $\chi_{p+q-2}^{2}$ sob ${H^{\prime}}^{\prime}$. Ainda, sob ${H^{\prime}}^{\prime}$, temos que $F=f I$, $H=h I, W=w I, \Phi=\phi I, \Phi_{1}=\delta_{1} I, \Phi_{2}=\delta_{2} I, \Phi_{3}=\delta_{3} I, X=S=1, Z_{2 \beta}=$ $(n \phi w)^{-1} 11^{T}, Z_{2 \beta d}=(n \phi w)^{-1} I, Z_{2 \beta}^{(2)}=(n \phi w)^{-2} 11^{T}, Z_{23}^{(3)}=Z_{2 \beta d} Z_{2 \beta} Z_{2 \beta d}=(n \phi w)^{-3} 11^{T}$, $Z_{2 \gamma}=2 \phi^{2}\left(n \delta_{1}^{2}\right)^{-1} 11^{T}, Z_{2 \gamma d}=2 \phi^{2}\left(n \delta_{1}^{2}\right)^{-1} I, Z_{2 \gamma}^{(3)}=Z_{2 \gamma d} Z_{2 \gamma} Z_{2 \gamma d}=8 \phi^{6}\left(n \delta_{1}^{2}\right)^{-3} 11^{T}$, onde $I$ é a matriz identidade de dimensão $n \times n, \delta_{1}=\partial \phi / \partial \tau, \delta_{2}=\partial^{2} \phi / \partial \tau^{2}, \delta_{3}=\left(2 \phi^{4}\right)^{-1}\left(-6 \delta_{1}^{4}+\right.$ $\left.4 \phi \delta_{1}^{2} \delta_{2}+\phi^{2} \delta_{2}^{2}\right)$. Definimos as matrizes $J=\left(j_{l m}\right)=X\left(X^{T} X^{\top}\right)^{-1} X^{T}$ e $N=\left(n_{l m}\right)=$ $S\left(S^{T} S\right)^{-1} S^{T}$, de forma que $Z_{\beta}=(\phi w)^{-1} J$ e $Z_{\gamma}=\left(2 \phi^{2} / \delta_{1}^{2}\right) N$. Além disto, temos $J_{d}=$ $\operatorname{diag}\left\{j_{11}, \ldots, j_{n n}\right\}$ e $N_{d}=\operatorname{diag}\left\{\mathrm{n}_{11}, \ldots, \mathrm{n}_{\mathrm{nn}}\right\}$.

De (2.11) obtemos, então,

$$
c=1+\frac{\varepsilon_{p+q}-\varepsilon_{2}}{p+q-2}
$$

onde

$$
\begin{gathered}
\varepsilon_{p+q}-\varepsilon_{2}=\quad \varepsilon_{\beta_{p}}-\varepsilon_{\beta_{1}}+\varepsilon_{\gamma_{p}}-\varepsilon_{\gamma_{1}}+\frac{\phi \delta_{2}}{\delta_{1}^{2}} \operatorname{tr}\left(J_{d} N_{d}\right) \\
-\frac{1}{2}\left\{2 \operatorname{tr}\left(J^{2} N\right)-1^{T} J_{d} V J_{d} 1\right\} \\
+\frac{2 \delta_{1}^{2}-\phi \delta_{2}}{\delta_{1}^{2}} 1^{T} J_{d} N N_{d} 1-\frac{3}{2 n}
\end{gathered}
$$

com

$$
\varepsilon_{\beta_{p}}-\varepsilon_{\beta_{1}}=\frac{h \phi}{4 \phi w^{2}} \operatorname{tr}\left(J_{d}^{2}\right)-\frac{f^{2}}{2 \phi w^{3}} 1^{T} J^{(3)} 1+\frac{f^{2}}{4 o w^{3}} 1^{T} J_{d} J J_{d} 1+\frac{f^{2}-h w}{4 n \phi w^{3}}
$$

e

$$
\begin{aligned}
\varepsilon_{\gamma q}-\varepsilon_{\gamma 1}= & \frac{1}{6 \delta_{1}^{4}}\left\{3\left(-6 \delta_{1}^{4}+4 \phi \delta_{1}^{2} \delta_{2}+\phi^{2} \delta_{2}^{2}\right) \operatorname{tr}\left(N_{d}^{2}\right)+2\left(4 \delta_{1}^{4}-3 \phi^{2} \delta_{2}^{2}\right) 1^{T} N^{(3)} 1\right. \\
& \left.+3\left(2 \delta_{1}^{2}-\phi \delta_{2}\right)^{2} 1^{T} N_{d} N N_{d} 1\right\}-\frac{1}{3 n} .
\end{aligned}
$$

Em alguns casos, dependendo das matrizes $X$ e $S$, as equações (2.14)a(2.16) podem ser bastante simplificadas. A equação (2.15) se anula para o modelo normal com funções de 
ligação identidade para a média ( $w=1, h=f=0$ ) e qualquer para o parâmetro de precisão. enquanto que as equações (2.14) e (2.16) não se alteram.

\subsubsection{Modelo de regressão linear simples}

Consideremos um modelo normal heterocedástico com componentes sistemáticas $d\left(\mu_{l}\right)=$ $\eta_{l}=\beta_{2}+\beta_{1} x_{l}$ e $g\left(\phi_{l}\right)=\tau_{l}=\gamma_{2}+\gamma_{1} s_{l}$, onde $x_{l}$ e $s_{l}$ denotam os valores das variáveis explicativas $x$ e $s$, para $l=1, \ldots, n$. Para o teste da hipótese composta $H^{\prime}{ }_{1}: \beta_{1}=0, \gamma_{1}=0$, a estatística da razão de verossimilhança é dada pela expressão $L R_{1}^{\prime}=2\left\{\ell\left(\hat{\beta}_{1}, \hat{\beta}_{2}, \hat{\gamma}_{1}, \hat{\gamma}_{2}\right)-\right.$ $\left.\ell\left(0, \tilde{\beta}_{2}, 0, \tilde{\gamma}_{2}\right)\right\}$. Neste caso, $p=q=2$ e a correção de Bartlett tem a forma $c=1+$ $\left(\varepsilon_{4}-\varepsilon_{2}\right) / 2$, onde a diferença $\varepsilon_{4}-\varepsilon_{2}$ vem das expressões (2.14) a (2.16). Definimos as notações $v_{a x}=\sum\left(x_{l}-\bar{x}\right)^{a} / n$ e $v_{a s}=\sum\left(s_{l}-\bar{s}\right)^{a} / n$ com $(a=2,3,4)$ para o $a$-ésimo momento central das covariáveis $x$ e $s$, onde $\bar{x}=\sum x_{l} / n$ e $\bar{s}=\sum s_{l} / n$. Definimos também, $v_{a x, b s}=\sum\left(x_{l}-\bar{x}\right)^{a}\left(s_{l}-\bar{s}\right)^{b} / n \operatorname{com}(a, b=1,2)$. Sob o modelo irrestrito temos $X=\left(\begin{array}{ll}1 & x\end{array}\right)$, $\operatorname{com} x=\left(x_{1}, \ldots, x_{n}\right)^{T}, S=\left(\begin{array}{ll}1 & s\end{array}\right), \operatorname{com} s=\left(s_{1}, \cdots, s_{n}\right)^{T}$ e os elementos $(l, m)$ das matrizes $Z_{\beta}$ e $Z \gamma$, avaliados em $\beta_{1}=\gamma_{1}=0$, dados por $z_{\beta_{l m}}=\left(n \phi w v_{2 x}\right)^{-1}\left\{v_{2 x}+\left(x_{l}-\bar{x}\right)\left(x_{m}-\bar{x}\right\}\right.$ e $z_{\gamma_{l m}}=\left[2 \phi^{2} /\left(n \delta_{1}^{2} v_{2 s}\right)\right]\left\{v_{2 s}+\left(s_{l}-\bar{s}\right)\left(s_{m}-\bar{s}\right)\right\}$, respectivamente. Utilizando a notação definida acima em conjunto com as fórmulas (2.14)a(2.16) encontramos

$$
\begin{aligned}
\varepsilon_{4}-\varepsilon_{2}= & \varepsilon_{\beta_{2}}-\varepsilon_{\beta_{1}}+\varepsilon_{\gamma_{2}}-\varepsilon_{\gamma_{1}}+\frac{1}{2 n \delta_{1}^{2}}\left\{\frac{2\left(\phi \delta_{2} v_{2 x, 2 s}-2 \delta_{1}^{2} v_{x, s}^{2}\right)}{v_{2 x} v_{2 s}}\right. \\
& \left.-\delta_{1}^{2} \frac{v_{2 x, s}^{2}}{v_{2 x}^{2} v_{2 s}}+2\left(2 \delta_{1}^{2}-\phi \delta_{2}\right) \frac{v_{2 x, s} v_{3 s}}{v_{2 x} v_{2 s}^{2}}+13 \delta_{1}^{2}-2 \phi \delta_{2}\right\},
\end{aligned}
$$

com

$$
\varepsilon_{\beta_{2}}-\varepsilon_{\beta_{1}}=\frac{1}{12 n \phi w^{3}}\left\{3\left(5 h w-3 f^{2}\right)+3 h w \gamma_{2 x}-3 f^{2} \gamma_{1 x}^{2}\right\}
$$

$\mathrm{e}$

$$
\varepsilon_{\gamma_{2}}-\varepsilon_{\gamma_{1}}=\frac{-1}{6 n \delta_{1}^{4}}\left[3\left\{-10 \phi^{4} \delta_{3}+3 \phi \delta_{2}\left(\phi \delta_{2}+4 \delta_{1}^{2}\right)-20 \delta_{1}^{4}\right\}\right.
$$




$$
\left.-6 \phi^{4} \delta_{3} \gamma_{2 s}+\left\{3 \phi \delta_{2}\left(\phi \delta_{2}+4 \delta_{1}^{2}\right)-20 \delta_{1}^{4}\right\} \gamma_{1 s}^{2}\right]
$$

onde $\gamma_{1 x}=v_{3 x} / v_{2 x}^{3 / 2}$ e $\gamma_{2 x}=v_{4 x} / v_{2 x}^{2}-3$ são as medidas amostrais usuais de assimetria e curtose padronizadas de $x$ e $\gamma_{1 s}=v_{3 s} / v_{2 s}^{3 / 2}$ e $\gamma_{2 s}=v_{4 s} / v_{2 s}^{2}-3$ são as medidas amostrais de assimetria e curtose padronizadas de $s$. Para $X=S$ as fórmulas (2.17) a (2.19) são expressas como

$$
\varepsilon_{4}-\varepsilon_{2}=\varepsilon_{\beta_{2}}-\varepsilon_{\beta_{1}}+\varepsilon_{\gamma_{2}}-\varepsilon_{\gamma_{1}}+\frac{1}{2 n \delta_{1}^{2}}\left\{2 \phi \delta_{2} \gamma_{2 x}+\left(3 \delta_{1}^{2}-2 \phi \delta_{2}\right) \gamma_{1 x}^{2}+9 \delta_{1}^{2}+4 \phi \delta_{2}\right\}
$$

com

$$
\varepsilon_{\beta_{2}}-\varepsilon_{\beta_{1}}=\frac{1}{12 n o w^{3}}\left\{3\left(5 h w-3 f^{2}\right)+3 h w \gamma_{2 x}^{2}-3 f^{2} \gamma_{1 x}^{2}\right\}
$$

e

$$
\begin{aligned}
\varepsilon_{\gamma_{2}}-\varepsilon_{\gamma_{1}}= & \frac{-1}{6 n \delta_{1}{ }^{4}}\left\{3\left[-10 \phi^{4} \delta_{3}+3 \phi \delta_{2}\left(\phi \delta_{2}+4 \delta_{1}{ }^{2}\right)-20 \delta_{1}{ }^{4}\right]\right. \\
& \left.-6 \phi^{4} \delta_{3} \gamma_{2 x}+\left[3 \phi \delta_{2}\left(\phi \delta_{2}+4 \delta_{1}{ }^{2}\right)-20 \delta_{1}{ }^{4}\right] \gamma_{1 x}^{2}\right\} .
\end{aligned}
$$

Para este caso, a alteração na expressão (2.19) consiste em substituir $\gamma_{2 s}$ e $\gamma_{1 s}^{2}$ por $\gamma_{2 x}$ e $\gamma_{1 x}^{2}$ respectivamente.

É fácil observar pelas equações $(2.17) a(2.19)$ que a aproximação da estatística $L R^{\prime}{ }_{1}$ para a distribuição $\chi_{2}^{2}$ é sensível a mudanças nas medidas amostrais de assimetria e curtose de $x$ e $s$. Para certos valores de $\gamma_{1 x}^{2}, \gamma_{1 s}^{2}, \gamma_{2 x}$ e $\gamma_{2 s}$ a estatística $L R_{1}^{\prime}$ pode ter aproximação de má qualidade para a distribuição $\chi_{2}^{2}$, o que sugere a necessidade da utilização da correção de Bartlett para amostras de tamanhos pequenos e moderados. No Capítulo 4 apresentamos alguns resultados de simulações onde analisamos a atuação dessa correção.

Trabalhando as expressões (2.17)a(2.19) para o modelo normal heterocedástico com funções de ligação identidade para $\mu(\mu=\eta=X \beta)$ e $\operatorname{logaritmica}$ para $\phi(-\log \phi=\tau=S \gamma)$, 
a equação (2.18) se anula, enquanto que as equações (2.17) e (2.19) resultam em

$$
\varepsilon_{4}-\varepsilon_{2}=\varepsilon_{\gamma_{2}}-\varepsilon_{\gamma_{1}}+\frac{1}{2 n v_{2 x}^{2} v_{2 s}^{2}}\left\{2\left(v_{2 x, 2 s}-2 v_{x, s}^{2}\right) v_{2 x} v_{2 s}+\left(2 v_{2 s} v_{3 s}-v_{2 s} v_{2 x, s}\right) v_{2 x, s}+11 v_{2 x}^{2} v_{2 s}^{2}\right\}
$$

e

$$
\varepsilon_{\gamma_{2}}-\varepsilon_{\gamma_{1}}=\frac{1}{6 n}\left(5 \gamma_{1 s}^{2}-3 \gamma_{2 s}\right)
$$

respectivamente. Neste caso, para $X=S$ temos

$$
\varepsilon_{4}-\varepsilon_{2}=\frac{1}{6 n}\left(8 \gamma_{1 x}^{2}+45\right)
$$

de modo que o fator de correção de Bartlett $c$ só depende da medida amostral de assimetria padronizada de $x$.

\subsection{Testes sobre o parâmetro de precisão}

Nesta Seção estamos interessados em testar hipóteses apenas sobre o parâmetro de precisão $\phi$, ou seja, sobre componentes do vetor $\gamma$. Consideremos, então, a partição $\gamma=$ $\left(\gamma_{1}^{T}, \gamma_{2}^{T}\right)^{T}$ para $\gamma$, onde $\gamma_{1}=\left(\gamma_{1}, \ldots \gamma_{q_{1}}\right)^{T}$ e $\gamma_{2}=\left(\gamma_{q_{1}+1}, \ldots \gamma_{q}\right)^{T}$ com $q_{1} \leq q$. Essa partição induz as seguintes partições $S=\left(S_{1}, S_{2}\right), \quad U=\left(U_{\beta}^{T}(\beta, \gamma), \quad U_{\gamma_{1}}^{T}\left(\beta, \gamma_{1}, \gamma_{2}\right), \quad U_{\gamma_{2}}^{T}\left(\beta, \gamma_{1}, \gamma_{2}\right)\right)^{T}$ e

$$
K_{\gamma, \gamma}=\left(\begin{array}{ll}
K_{\gamma_{11}} & K_{\gamma_{12}} \\
K_{\gamma_{21}} & K_{\gamma_{22}}
\end{array}\right),
$$

onde as matrizes $S_{1}, S_{2}$ e $K_{\gamma_{i j}}$, para $i, j=1,2$, estão definidas na Seção 2.2 .

A estatística da razão de verossimilhança para testar $H_{2}: \gamma_{1}=\gamma_{1}^{(0)}$ contra $H: \gamma_{1} \neq \gamma_{1}^{(0)}$, onde $\gamma_{1}^{(0)}$ é um vetor especificado de dimensão $q_{1}$ e $\beta$ e $\gamma_{2}$ são vetores de parâmetros de perturbação, é dada por

$$
L R_{2}=2\left\{\ell\left(\widehat{\beta}, \widehat{\gamma}_{1}, \hat{\gamma}_{2}\right)-\ell\left(\tilde{\beta}, \gamma_{1}^{(0)}, \tilde{\gamma}_{2}\right)\right\}
$$


A distribuição de $L R_{2}$ sob $H_{2}$ e para grandes amostras é $\chi_{q_{1}}^{2}$. A correção de Bartlett para $L R_{2}$ tem a forma $c=q_{1}^{-1} E\left(L R_{2}\right)$, onde o termo $E\left(L R_{2}\right)$ é calculado até ordem $O\left(n^{-1}\right)$, sob $H_{2}$. Reescrevendo $L R_{2}$ como

$$
L R_{2}=2\left[\left\{\ell\left(\widehat{\beta}, \widehat{\gamma}_{1}, \hat{\gamma}_{2}\right)-\ell\left(\beta, \gamma_{1}^{(0)}, \gamma_{2}\right)\right\}-\left\{\ell\left(\tilde{\beta}, \gamma_{1}^{(0)}, \tilde{\gamma}_{2}\right)-\ell\left(\beta, \gamma_{1}^{(0)}, \gamma_{2}\right)\right\}\right],
$$

onde $\ell\left(\beta, \gamma_{1}^{(0)}, \gamma_{2}\right)$ é o logaritmo da função de verossimilhança calculada nos parâmetros e usando os resultados (2.1) a (2.4) em conjunto com os de Botter e Cordeiro (1997) obtemos que, até ordem $O\left(n^{-1}\right), E\left\{2\left[\ell\left(\widehat{\beta}, \widehat{\gamma}_{1}, \widehat{\gamma}_{2}\right)-\ell\left(\beta, \gamma_{1}^{(0)}, \gamma_{2}\right)\right]\right\}=p+q+\varepsilon_{p+q}$ e $2 \mathrm{E}\left\{\ell\left(\tilde{\beta}, \gamma_{1}^{(0)}, \tilde{\gamma}_{2}\right)-\right.$ $\left.\ell\left(\beta, \gamma_{1}^{(0)}, \gamma_{2}\right)\right\}=p+\left(q-q_{1}\right)-\varepsilon_{p+\left(q-q_{1}\right)}$. Até ordem $O\left(n^{-1}\right)$, temos, então, que

$$
\mathrm{E}\left(L R_{2}\right)=q_{1}+\varepsilon_{p+q}-\varepsilon_{p+\left(q-q_{1}\right)}
$$

onde

$$
\begin{aligned}
\varepsilon_{p+q}-\varepsilon_{p+\left(q-q_{1}\right)}= & \varepsilon_{\gamma q}-\varepsilon_{\gamma\left(q-q_{1}\right)}+\frac{1}{2} \operatorname{tr}\left\{\Phi_{2} W Z_{\beta d}\left(Z_{\gamma d}-Z_{2 \gamma d}\right)\right. \\
& \left.-\Phi_{1} W Z_{\beta}^{(2)} W \Phi_{1}\left(Z_{\gamma}-Z_{2 \gamma}\right)\right\}+\frac{1}{4} 1^{T} \Phi_{1} W Z_{\beta d} \\
& \left(Z_{\gamma}-Z_{2 \gamma}\right) Z_{\beta d} W \Phi_{1} 1+\frac{1}{4} 1^{T} \Phi_{1} W Z_{\beta d}\left(Z_{\gamma} Z_{\gamma d}-Z_{2 \gamma} Z_{2 \gamma d}\right) \\
& \left(2 \Phi^{3} \Phi_{1}^{2}+\Phi^{2} \Phi_{2}\right) \Phi_{1} 1
\end{aligned}
$$

$\operatorname{com} \varepsilon_{\gamma q}-\varepsilon_{\gamma\left(q-q_{1}\right)}$ dado por (2.10) e as matrizes $Z_{2 \gamma}$ e $Z_{2 \gamma d}$ assumindo as mesmas expressões daquelas definidas após (2.10). Como na Seção 2.1, todas as matrizes diagonais $W, \Phi, \Phi_{1}$ e $\Phi_{2}$ devem ser calculadas sob $H_{2}$.

A correção de Bartlett associada a $L R_{2}$ é dada por

$$
c=1+\frac{\varepsilon_{p+q}-\varepsilon_{p+\left(q-q_{1}\right)}}{q_{1}},
$$


onde $\varepsilon_{p+q}-\varepsilon_{p+\left(q-q_{1}\right)}$ é obtida de (2.20). A estatística corrigida $L R_{2}^{*}=\tilde{c}^{-1} L R_{2}$ tem, sob $H_{2}$, distribuição $\chi_{q_{1}}^{2}$ até $O\left(n^{-1}\right)$.

Para o modelo normal introduzido na Seção 1.2, considerando função de ligação identidade para a média $(\mu=\eta=X \beta)$ e logaritmica para o parâmetro de precisão $(-\log \phi=\tau=$ $S \gamma)$, a correção de Bartlett se reduz a

$$
\begin{aligned}
c= & \frac{1}{q_{1}}\left[-\frac{1}{8} \operatorname{tr}\left(Z_{\gamma d}^{2}-Z_{2 \gamma d}^{2}\right)+\frac{1}{24} 1^{T}\left(Z_{\gamma}^{(3)}-Z_{2 \gamma}^{(3)}\right) 1+\frac{1}{16} 1^{T}\left(Z_{\gamma d} Z_{\gamma} Z_{\gamma d}\right)\right. \\
& -\left(Z_{2 \gamma d} Z_{2 \gamma} Z_{2 \gamma d}\right) 1+\frac{1}{2} \operatorname{tr}\left\{\Phi^{-1} Z_{\beta d}\left(Z_{\gamma d}-Z_{2 \gamma d}\right)-\Phi^{-1} Z_{\beta}^{(2)} \Phi^{-1}\left(Z_{\gamma}-Z_{2 \gamma}\right)\right\} \\
& \left.+\frac{1}{4} 1^{T} \Phi^{-1} Z_{\beta d}\left(Z_{\gamma}-Z_{2 \gamma}\right) Z_{\beta d} \Phi^{-1} 1+\frac{1}{4} 1^{T} \Phi^{-1} Z_{\beta d}\left(Z_{\gamma} Z_{\gamma d}-Z_{2 \gamma} Z_{2 \gamma d}\right) 1\right] .
\end{aligned}
$$

A estatística da razão de verossimilhança para o teste $H_{2}^{\prime}: \gamma_{1}=0$ contra $H: \gamma_{1} \neq 0$, assumindo que $s_{l q}=1$, para $l=1, \ldots, n$, onde $\gamma_{q}$ e $\beta$ são parâmetros de perturbação, reduz-se a

$$
L R_{2}^{\prime}=2\left\{\ell\left(\widehat{\beta}, \widehat{\gamma}_{1}, \widehat{\gamma}_{q}\right)-\ell\left(\tilde{\beta}, 0, \tilde{\gamma}_{q}\right)\right\}
$$

com distribuição assintótica $\chi_{q-1}^{2}$, sob ${H^{\prime}}_{2}$. A correção de Bartlett obtida de (2.21) é dada por

$$
c=1+\frac{\varepsilon_{p+q}-\varepsilon_{p+1}}{q-1}
$$

com

$$
\begin{aligned}
\varepsilon_{p+q}-\varepsilon_{p+1}= & \varepsilon_{\gamma_{q}}-\varepsilon_{\gamma_{1}}+\frac{\phi^{2}}{\delta_{1}^{2}} \operatorname{tr}\left(\delta_{2} W Z_{\beta d} N_{d}-\delta_{1}^{2} W Z_{\beta}^{(2)} W N\right) \\
& +\frac{\phi^{2}}{2} 1^{T} W Z_{\beta d} N\left\{Z_{\beta d} W+\left(4 \phi^{-1}-2 \delta_{1}^{-2}\right) N_{d}\right\} 1 \\
& -\frac{p(p+2)}{2 n}
\end{aligned}
$$


onde $\varepsilon_{\gamma_{q}}-\varepsilon_{\gamma_{1}}$ se reduz à expressão (2.16). A estatística corrigida $L R_{2}^{\prime *}=\tilde{c}^{-1} L R_{2}^{\prime}$ tem, sob $H^{\prime}{ }_{2}$ e até ordem $O\left(n^{-1}\right)$, a distribuição $\chi_{q-1}^{2}$.

Como aplicação para o teste $H^{\prime}{ }_{2}$ consideremos o modelo normal com função de ligação identidade para a média $(\mu=\eta=X \beta)$ e função de ligação qualquer para o parâmetro de precisão. Temos $W=I$. De (2.23) obtemos

$$
\begin{aligned}
\varepsilon_{p+q}-\varepsilon_{p+1}= & \varepsilon_{\gamma_{q}}-\varepsilon_{\gamma_{1}}+\frac{1}{\delta_{1}^{2}} \operatorname{tr}\left(\phi \delta_{2} J_{d} N_{d}-\delta_{1}^{2} J^{(2)} N\right) \\
& +\frac{1}{2 \delta_{1}^{2}} 1^{T} J_{d} N\left\{\delta_{1}^{2} J_{d}+2\left(2 \delta_{1}^{2}-\phi \delta_{2}\right) N_{d}\right\} 1 \\
& -\frac{p(p+2)}{2 n}
\end{aligned}
$$

onde $\varepsilon_{\gamma_{q}}-\varepsilon_{\gamma_{1}}$ é dado por (2.16).

Ao considerar, no teste anterior, que a função de ligação para o parâmetro de precisão é a logaritmica, isto é, $(-\log \phi=\tau=S \gamma)$, o termo $\varepsilon_{p+q}-\varepsilon_{p+1}$ em (2.23) se reduz a

$$
\begin{aligned}
\varepsilon_{p+q}-\varepsilon_{p+1}= & \operatorname{tr}\left(J_{d} N_{d}-J^{(2)} N\right)+\frac{1}{2} 1^{T} J_{d} N\left(J_{d}+2 N_{d}\right) 1-\frac{1}{2} \operatorname{tr}\left(N_{d}^{2}\right) \\
& +\frac{1}{3} 1^{T} N^{(3)} 1+\frac{1}{2} 1^{T} N-d N N_{d} 1-\frac{3(p+1)^{2}-1}{6 n} .
\end{aligned}
$$

\subsubsection{Modelo de regressão linear simples}

Consideremos o modelo de regressão linear simples para $\mu$ como $d\left(\mu_{l}\right)=\eta_{l}=\beta_{2}+\beta_{1} x_{l}$ e para $\phi$ como $g\left(\phi_{l}\right)=\tau_{l}=\gamma_{2}+\gamma_{1} s_{l}$, onde $x_{l}$ e $s_{l}$ são os valores das variáveis explicativas associadas a $y_{l}$, para $l=1, \ldots, n$. Para o teste de ${H^{\prime}}_{2}: \gamma_{1}=0$ contra $H: \gamma_{1} \neq 0$ a estatística da razão de verossimilhança tem a forma $L R_{2}^{\prime}=2\left\{\ell\left(\hat{\beta}, \hat{\gamma}_{2}, \hat{\gamma}_{1}\right)-\ell\left(\tilde{\beta}, \tilde{\gamma}_{2}, 0\right)\right\}$ e a correção de Bartlett é dada por $c=1+\varepsilon_{4}-\varepsilon_{3}$, onde a expressão $\varepsilon_{4}-\varepsilon_{3}$ é obtida de (2.23), com o termo 
$\varepsilon_{\gamma_{2}}-\varepsilon_{\gamma_{1}}$ dado em (2.19), lembrando que sob a hipótese nula o $(l, m)$-ésimo elemento de $Z_{\gamma}$ é igual a $z_{\gamma_{l m}}=2 \phi^{2}\left(n \delta_{1}^{2} v_{2 s}\right)^{-1}\left\{v_{2 s}+\left(s_{l}-\bar{s}\right)\left(s_{m}-\bar{s}\right)\right\}$.

Como aplicações, consideremos os três casos a seguir. Como primeiro caso, assumimos $\mu=\eta=X \beta$, ou seja, função de ligação identidade para a média e qualquer para o parâmetro de precisão. Temos, neste caso, $W=I$ e sob a hipótese nula $H_{2}{ }^{\prime}$, o $(l, m)$-ésimo elemento de $Z_{\beta}$ é igual a $z_{\beta_{l m}}=\left(n \phi v_{2 x}\right)^{-1}\left\{v_{2 x}+\left(x_{l}-\bar{x}\right)\left(x_{m}-\bar{x}\right)\right\}$. Logo, $\varepsilon_{4}-\varepsilon_{3}$ é dado por

$$
\begin{aligned}
\varepsilon_{4}-\varepsilon_{3}= & \varepsilon_{\gamma_{2}}-\varepsilon_{\gamma_{1}}-\frac{v_{2 x, s}^{2}+4 v_{x, s}^{2} v_{2 x}}{2 n v_{2 x}^{2} v_{2 s}} \\
& +\frac{\phi \delta_{2} v_{2 x, 2 s} v_{2 s}+\left(2 \delta_{1}^{2}-\phi \delta_{2}\right) v_{2 x, s} v_{3 s}}{n \delta_{1}^{2} v_{2 x} v_{2 s}^{2}} \\
& +\frac{8 \delta_{1}^{2}-\phi \delta_{2}}{n \delta_{1}^{2}}-\frac{p(p+2)}{2 n}
\end{aligned}
$$

$\operatorname{com} \varepsilon_{\gamma_{2}}-\varepsilon_{\gamma_{1}}$ dado por (2.19).

Para um segundo caso, adotamos $\mu=\eta=X \beta$ e $-\log \phi=\tau=S \gamma$ (função de ligação identidade para a média e logaritmica para o parâmetro de precisão). Logo, $\varepsilon_{4}-\varepsilon_{3}$ é dado por

$$
\begin{aligned}
\varepsilon_{4}-\varepsilon_{3}= & \varepsilon_{\gamma_{2}}-\varepsilon_{\gamma_{1}}-\frac{v_{2 x, s}^{2}+4 v_{x, s}^{2} v_{2 x}}{2 n v_{2 x}^{2} v_{2 s}} \\
& +\frac{v_{2 x, 2 s} v_{2 s}+v_{2 x, s} v_{3 s}}{n v_{2 x} v_{2 s}^{2}} \\
& +\frac{14-p(p+2)}{2 n}
\end{aligned}
$$

$\operatorname{com} \varepsilon_{\gamma_{2}}-\varepsilon_{\gamma_{1}}$ dado por

$$
\varepsilon_{\gamma_{2}}-\varepsilon_{\gamma_{1}}=\frac{1}{6 n}\left(5 \gamma_{1 s}^{2}-3 \gamma_{2 s}\right)
$$

com $\gamma_{1 s}^{2}$ e $\gamma_{2 s}$ como definidos anteriormente. 
No terceiro caso, consideramos $\mu=\eta=X \beta$ e $X=S$ (função de ligação identidade para a média e qualquer para o parâmetro de precisão e as matrizes X e S idênticas). Logo, $\varepsilon_{4}-\varepsilon_{3}$ é dado por

$$
\begin{aligned}
\varepsilon_{4}-\varepsilon_{3}= & \varepsilon_{\gamma_{2}}-\varepsilon_{\gamma_{1}}+\frac{\phi \delta_{2}}{n \delta_{1}^{2}} \gamma_{2 x} \\
& +\frac{3 \delta_{1}^{2}-2 \phi \delta_{2} \phi \delta}{2 n \delta_{1}^{2}} \gamma_{1 x}^{2} \\
& +\frac{6 \delta_{1}^{2}+2 \phi \delta_{2}}{n \delta_{1}^{2}}-\frac{p(p+2)}{2 n}
\end{aligned}
$$

com

$$
\begin{aligned}
\varepsilon_{\gamma_{2}}-\varepsilon_{\gamma_{1}}= & \frac{-1}{6 n \delta_{1}^{4}}\left[3\left\{-10 \phi^{4} \delta_{3}+3 \phi \delta_{2}\left(\phi \delta_{2}+4 \delta_{1}^{2}\right)-20 \delta_{1}^{4}\right\}\right. \\
& \left.-6 \phi^{4} \delta_{3} \gamma_{2 x}+\left\{3 \phi \delta_{2}\left(\phi \delta_{2}+4 \delta_{1}^{2}\right)-20 \delta_{1}^{4}\right\} \gamma_{1 x}^{2}\right] .
\end{aligned}
$$

\subsection{Testes sobre a média}

Suponhamos agora, que nosso interesse seja testar hipóteses apenas sobre a média em um modelo normal heterocedástico dado por (1.1), isto é, sobre componentes do vetor $\beta$. Consideremos então, a partição $\beta=\left(\beta_{1}^{T}, \beta_{2}^{T}\right)^{T}$, onde $\beta_{1}=\left(\beta_{1}, \ldots \beta_{p_{1}}\right)^{T}$ e $\beta_{2}=\left(\beta_{p_{1}+1}\right.$, $\left.\ldots, \beta_{p}\right)^{T} \operatorname{com} p_{1} \leq p$. Essa partição induz as correspondentes partições $X=\left(X_{1}, X_{2}\right)$, $U=\left(U_{\beta_{1}}^{T}\left(\beta_{1}, \beta_{2}, \gamma\right), U_{\beta_{2}}^{T}\left(\beta_{1}, \beta_{2}, \gamma\right), U_{\gamma}^{T}\left(\beta_{1}, \beta_{2}, \gamma\right)\right)^{T}$ e

$$
K_{\beta, \beta}=\left(\begin{array}{ll}
K_{\beta_{11}} & K_{\beta_{12}} \\
K_{\beta_{21}} & K_{\beta_{22}}
\end{array}\right),
$$

onde $X_{1}, X_{2}$ e $K_{\beta_{i}}$ para $i, j=1,2$ foram definidos na Seção 2.2 .

Estamos interessados em testar a hipótese $H_{3}: \beta_{1}=\beta_{1}^{(0)}$ contra $H: \beta_{1} \neq \beta_{1}^{(0)}$, onde $\beta_{1}^{(0)}$ é um vetor especificado de dimensão $p_{1}$ e $\beta_{2}$ e $\gamma$ são vetores de parâmetros de perturbação. 
A estatística da razão de verossimilhança para testar $H_{3}$ é dada por

$$
L R_{3}=2\left\{\ell\left(\widehat{\beta}_{1}, \hat{\beta}_{2}, \hat{\gamma}\right)-\ell\left(\beta_{1}^{(0)}, \tilde{\beta}_{2}, \tilde{\gamma}\right)\right\}
$$

que sob $H_{3}$, tem distribuição assintótica $\chi_{p_{1}}^{2}$. Reescrevendo

$$
L R_{3}=2\left[\left\{\ell\left(\widehat{\beta}_{1}, \widehat{\beta}_{2}, \widehat{\gamma}\right)-\ell\left(\beta_{1}^{(0)}, \beta_{2}, \gamma\right)\right\}-\left\{\ell\left(\beta_{1}^{(0)}, \tilde{\beta}_{2}, \tilde{\gamma}\right)-\ell\left(\beta_{1}^{(0)}, \beta_{2}, \gamma\right)\right\}\right],
$$

onde $\ell\left(\beta_{1}^{(0)}, \beta_{2}, \gamma\right)$ é o logaritmo da função de verossimilhança calculada nos parâmetros e usando os resultados (2.1) a (2.4) em conjunto com os de Cordeiro e Botter (1997) temos, até ordem $O\left(n^{-1}\right)$ que, $2 E\left\{\ell\left(\widehat{\beta}_{1}, \hat{3}_{2}, \widehat{\gamma}\right)-\ell\left(\beta_{1}^{(0)}, \beta_{2}, \gamma\right)\right\}=p+q+\varepsilon_{p+q}$ e $2 E\left\{\ell\left(\beta_{1}^{(0)}, \tilde{\beta}_{2}, \tilde{\gamma}\right)-\right.$ $\left.\ell\left(\beta_{1}^{(0)}, \beta_{2}, \gamma\right)\right\}=\left(p-p_{1}\right)+q-\varepsilon_{\left(p-p_{1}\right)+q}$. Até ordem $O\left(n^{-1}\right)$, temos, então, que

$$
E\left(L R_{3}\right)=p_{1}+\varepsilon_{p+q}-\varepsilon_{\left(p-p_{1}\right)+q},
$$

onde

$$
\begin{aligned}
\varepsilon_{p+q}-\varepsilon_{\left(p-p_{1}\right)+q}= & \varepsilon_{\beta p}-\Sigma_{3\left(p-p_{1}\right)}+\frac{1}{2} \operatorname{tr}\left\{\Phi_{2} W\left(Z_{\beta d}-Z_{2 \beta d}\right) Z_{\gamma d}\right. \\
& \left.-\Phi_{1} I^{\prime}\left(Z_{\beta}^{(2)}-Z_{2 \beta}^{(2)}\right) \Phi_{1} W Z_{\gamma}\right\}+\frac{1}{4} 1^{T} \Phi_{1} W\left(Z_{\beta d} Z_{\gamma} Z_{\beta d}\right. \\
& \left.-Z_{2 \beta d} Z_{\gamma} Z_{2 \beta d}\right) W \Phi_{1} 1+\frac{1}{4} 1^{T} \Phi_{1} W \\
& \left(Z_{\beta d}-Z_{2 \beta d}\right) Z_{\gamma} Z_{\gamma d}\left(2 \Phi^{-3} \Phi_{1}^{2}-\Phi^{-2} \Phi_{2}\right) \Phi_{1} 1
\end{aligned}
$$

$\operatorname{com} \varepsilon_{\beta p}-\varepsilon_{\beta\left(p-p_{1}\right)}$ dado por (2.9). Aqui, todas as matrizes diagonais $W, F, G, H, \Phi, \Phi_{1}$ e $\Phi_{2}$ devem ser avaliadas sob a hipótese nula $\mathrm{H}_{3}$.

A correção de Bartlett associada com $L R_{3}$ é $c=p_{1}^{-1} E\left(L R_{3}\right)$, onde $E\left(L R_{3}\right)$ vem de (2.34) e assume a forma

$$
c=1+\frac{\varepsilon_{p+q}-\varepsilon_{\left(p-p_{1}\right)+q}}{p_{1}}
$$


onde $\varepsilon_{p+q}-\varepsilon_{\left(p-p_{1}\right)+q}$ é dado por (2.35). Sob $H_{3}$, a estatística corrigida $L R_{3}^{*}=\tilde{c}^{-1} L R_{3}$ é distribuída até ordem $O\left(n^{-1}\right)$ como $\chi_{p_{1}}^{2}$.

Como aplicação de (2.36), consideremos o modelo normal com função de ligação identidade, $\mu=\eta=X \beta$, para a média e, logaritmica, $-\log \phi=\tau=S \gamma$, para o parâmetro de precisão. O termo $\varepsilon_{\beta p}-\varepsilon_{\beta\left(p-p_{1}\right)}$ em (2.35) se anula e a correção de Bartlett $c$ em (2.36) se reduz a

$$
\begin{aligned}
c= & +\frac{1}{p_{1}}\left[\frac{1}{2} \operatorname{tr}\left\{\Phi\left(Z_{\beta d}-Z_{2 \beta d}\right) Z_{\gamma d}-\Phi\left(Z_{\beta}^{(2)}-Z_{2 \beta}^{(2)}\right) \Phi Z_{\gamma}\right\}\right. \\
& \left.+\frac{1}{4} 1^{T} \Phi\left(Z_{\beta d} Z_{\gamma} Z_{\beta d}-Z_{2 \beta d} Z_{\gamma} Z_{2 \beta d}\right) \Phi 1+\frac{1}{4} 1^{T} \Phi\left(Z_{\beta d}-Z_{2 \beta d}\right) Z_{\gamma} Z_{\gamma d} 1\right]
\end{aligned}
$$

que se iguala à correção de Bartlett $c_{3}$ obtida por Cordeiro (1993, página 179).

Se $q=1$ e $S=1$, as matrizes diagonais $\Phi^{-1}, \Phi_{1}$ e $\Phi_{2}$ em (2.35), são as mesmas definidas para testar a hipótese nula $H_{1}$ na Secão $2.2, Z_{\gamma}=2 \phi^{2}\left(n \delta_{1}^{2}\right)^{-1} 11^{T}$ e $Z_{\gamma d}=2 \phi^{2}\left(n \delta_{1}^{2}\right)^{-1} I$. A correção de Bartlett toma a forma

$$
c=1+\frac{\varepsilon_{p+1}-\varepsilon_{\left(p-p_{1}\right)+1}}{p_{1}}
$$

com

$$
\varepsilon_{p+1}-\varepsilon_{\left(p-p_{1}\right)+1}=\varepsilon_{\beta p}-\varepsilon_{\beta\left(p-p_{1}\right)}+\frac{p_{1}\left(4-p_{1}\right)}{2 n}
$$

e

$$
\begin{aligned}
\varepsilon_{\beta p}-\varepsilon_{\beta\left(p-p_{1}\right)}= & \frac{\phi}{4} \operatorname{tr}\left\{H\left(Z_{\beta d}^{2}-Z_{2 \beta d}^{2}\right)\right\}-\frac{\phi^{2}}{2} 1^{T} F\left(Z_{\beta}^{(3)}-Z_{2 \beta}^{(3)}\right) F 1 \\
& +\frac{\phi^{2}}{4} 1^{T} F\left(Z_{\beta d} Z_{\beta} Z_{\beta d}-Z_{2 \beta d} Z_{2 \beta} Z_{2 \beta d}\right) F 1 .
\end{aligned}
$$

Considerando ainda $q=1$ e $S=1$, a estatística da razão de verossimilhança para testar a hipótese $H^{\prime}{ }_{3}: \beta_{1}=0$ contra $H: \beta_{1} \neq 0$, assumindo que $p_{1}=p-1$ e $x_{l p}=1$, para $l=1, \ldots, n$, é dada por

$$
L R^{\prime}{ }_{3}=2\left\{\ell\left(\widehat{\beta}_{1}, \widehat{\beta}_{2}, \widehat{\gamma}\right)-\ell\left(0, \tilde{\beta}_{2}, \tilde{\gamma}\right)\right\}
$$


que sob $H_{3}{ }^{\prime}$ é assintóticamente distribuída como $\chi_{p-1}^{2}$. As matrizes $W, F, H, \Phi, \Phi_{1}$. $\Phi_{2}, Z_{2 B}$, $Z_{2 \beta d}, Z_{23}^{(2)} . Z_{23}^{(3)}$ são definidas como as do teste de $H_{1}$ na Seção 2.2. De (2.36) segue que

$$
c=1+\frac{\varepsilon_{p+1}-\varepsilon_{2}}{p-1}
$$

com

$$
\varepsilon_{p+1}-\varepsilon_{2}=\varepsilon_{\beta p}-\varepsilon_{\beta 1}+\frac{(p-1)(p+3)}{2 n}
$$

e

$$
\begin{aligned}
\varepsilon_{\beta p}-\varepsilon_{\beta 1}= & \frac{\phi h}{4} \operatorname{tr}\left(Z_{\beta d}^{2}\right)-\frac{\phi^{2} f^{2}}{2} 1^{T} Z_{\beta}^{(3)} 1 \\
& +\frac{\phi^{2} f^{2}}{4} 1^{T} Z_{\beta d} Z_{\beta} Z_{\beta d} 1+\frac{f^{2}-h w}{4 n w^{3} \phi} .
\end{aligned}
$$

Para o modelo de regressão introduzido na Seção 2.2.1 com funções de ligação identidade para a média e logaritmica para o parâmetro de precisão, respectivamente, a correção de Bartlett $c$ para o teste de $\beta_{1}=0$ vem de (2.37) apresentando pouca simplificação. 


\section{Capítulo 3}

\section{Correção tipo-Bartlett para a}

\section{estatística escore}

\subsection{Introdução}

O teste escore, assintoticamente equivalente ao teste da razão de verossimilhança (White, 1984), requer somente estimação sob a hipótese nula, o que o torna mais atraente quando a estimação sob a hipótese alternativa exige um extenso trabalho computacional.

Harris (1985) obteve uma expansão assintótica para a distribuição da estatística escore $S_{R}$, até ordem $n^{-1}$. Em lugar de modificar a estatística escore, Harris mostrou que até ordem $O\left(n^{-1}\right), P\left(S_{R} \leq z_{\alpha}\right)=P\left(\chi^{2} \leq x_{\alpha}\right)=1-\alpha$, onde $z_{\alpha}$ é o quantil modificado de $S_{R}$ e $x_{\alpha}$ é o quantil da distribuição $\backslash^{2}$ de referência associados a um nível de significância $\alpha$. Honda (1988) derivou a correção do valor crítico da estatística $S_{R}$ para o teste de homocedasticidade no modelo definido em (1.1) considerando função de ligação identidade para a média e qualquer para o parâmetro de precisão. 
Baseados no trabalho de Harris (1985), Cordeiro e Ferrari (1991) obtiveram um fator de correção para $S_{R}$, denominado fator de correção tipo-Bartlett, de forma que a estatística escore aperfeiçoada por esse fator de correção tem distribuição $\chi^{2}$ até ordem $n^{-1}$ sob a hipótese nula. Segundo eles, sob certas condições de regularidade, a estatística escore modificada é obtida através da multiplicação da estatística escore original por um polinômio de segundo grau na própria estatística.

A partir do trabalho de Cordeiro e Ferrari (1991), muitos resultados têm sido publicados envolvendo estatísticas escore aperfeiçoadas por fatores de correção tipo-Bartlett em várias classes de modelos de regressão. Cribari-Neto e Ferrari (1995a, b) apresentam correções tipoBartlett para a estatística escore em teste de hipóteses no modelo normal heterocedástico definido em (1.1), considerando apenas a função de ligação identidade para a média. Neste capítulo, encontramos correções do tipo-Bartlett para a estatística escore associada a testes de hipóteses sobre a média e/ou parâmetro de precisão em modelos normais heterocedásticos com funções de ligação quaisquer para a média e para o parâmetro de precisão.

Neste trabalho não consideramos modificações no valor crítico da estatística escore ou qualquer estudo sobre a monotonicidade desta estatística, como sugerido em Cordeiro, Ferrari e Cysneiros(1998). Nos restringimos apenas a estudos de correções na própria estatí stica escore.

\subsection{Testes simultâneos sobre a média e o parâmetro de precisão}

Nessa seção, obtemos correções tipo-Bartlett para as estatísticas escore dos testes das hipóteses sobre a média e o parâmetro de precisão simultaneamente. Consideremos o modelo 
normal definido em (1.1) e as partições $\beta^{T}=\left(\beta_{1}{ }^{T}, \beta_{2}{ }^{T}\right)$ para o vetor $\beta$ e $\gamma^{T}=\left(\gamma_{1}{ }^{T}, \gamma_{2}{ }^{T}\right)$ para o vetor $\gamma$, onde $\beta_{1}=\left(\beta_{1} \ldots, \beta_{p_{1}}\right)^{T}, \beta_{2}=\left(\beta_{p_{1}+1}, \ldots, \beta_{p}\right)^{T}, \gamma_{1}=\left(\gamma_{1}, \ldots, \gamma_{q_{1}}\right)^{T}$ e $\gamma_{2}=\left(\gamma_{q_{1}+1}, \ldots, \gamma_{q}\right)^{T}$, com $p_{1} \leq p$ e $q_{1} \leq q$. Essas decomposições induzem as correspondentes partições $X=\left(X_{1}, X_{2}\right)$ e $S=\left(S_{1}, S_{2}\right)$, onde $X_{1}, X_{2}, S_{1}$ e $S_{2}$ são matrizes conhecidas de posto completo e dimensões $n \times p_{1}, n \times\left(p-p_{1}\right), n \times q_{1}, n \times\left(q-q_{1}\right)$, respectivamente.

Estamos interessados em testar a hipótese $H_{1}: \beta_{1}=\beta_{1}{ }^{(0)}, \gamma_{1}=\gamma_{1}{ }^{(0)}$ contra $H$ : pelo menos uma das igualdades é violada, onde $\beta_{1}{ }^{\left({ }^{0}\right)} \mathrm{e} \gamma_{1}{ }^{(0)}$ são vetores conhecidos de dimensões $p_{1}$ e $q_{1}$, respectivamente.

Seja $\theta_{1}=\left(\beta_{1}^{T}, \gamma_{1}^{T}\right)^{T}$ o vetor de parâmetros de interesse e $\theta_{2}=\left(\beta_{2}^{T}, \gamma_{2}^{T}\right)^{T}$ o vetor de parâmetros de perturbação. A função escore correspondente é dada por

$$
U=\left(U_{1}^{T}, U_{2}^{T}\right)^{T}
$$

onde

$$
U_{1}^{T}=\partial \ell(\beta, \gamma) / \partial \theta_{1}=\left(U_{\beta_{1}}^{T}\left(\beta_{1}, \beta_{2}, \gamma_{1}, \gamma_{2}\right), \quad U_{\gamma_{1}}^{T}\left(\beta_{1}, \beta_{2}, \gamma_{1}, \gamma_{2}\right)\right)^{T}
$$

e

$$
U_{2}^{T}=\partial \ell(\beta, \gamma) / \partial \theta_{2}=\left(U_{\beta_{2}}^{T}\left(\beta_{1}, \beta_{2}, \gamma_{1}, \gamma_{2}\right), \quad U_{\gamma_{2}}^{T}\left(\beta_{1}, \beta_{2}, \gamma_{1}, \gamma_{2}\right)\right)^{T}
$$

Além disto, a matriz de informação total de Fisher correspondente é dada por

$$
K=\left(\begin{array}{ll}
K_{11} & K_{12} \\
K_{21} & K_{22}
\end{array}\right)=\left(\begin{array}{cc:cc}
K_{\beta_{11}} & 0 & K_{\beta_{12}} & 0 \\
0 & K_{\gamma_{11}} & 0 & K_{\gamma_{12}} \\
\hdashline K_{\beta_{21}} & 0 & K_{\beta_{22}} & 0 \\
0 & K_{\gamma_{21}} & 0 & K_{\gamma_{22}}
\end{array}\right)
$$

onde as matrizes $K_{\beta_{11}}, K_{\beta_{12}}=K_{\beta_{21}}^{T}, K_{\beta_{22}}, K_{\gamma_{11}}, K_{\gamma_{12}}=K_{\gamma_{21}}^{k T}$ e $K_{\gamma_{22}}$ estão definidas na Seção 2.2. Definimos, também, as matrizes

$$
A=\left(\begin{array}{cc}
0 & 0 \\
0 & K_{22}^{-1}
\end{array}\right)
$$


e $M=K^{-1}-A$, onde

$$
K^{-1}=\left(\begin{array}{ll}
K^{11} & K^{12} \\
K^{21} & K^{22}
\end{array}\right)
$$

De acordo com Rao (1948), a estatística escore $S_{R}$ para o teste de $H_{1}$ tem a forma

$$
S_{R}=\tilde{U}_{1}^{T} \tilde{K}^{11} \tilde{U}_{1}
$$

ou seja, $U_{1}$ e $K^{11}$ são avaliadas em $(\tilde{\beta}, \tilde{\gamma})=\left(\beta_{1}^{(0)^{T}}, \tilde{\beta}_{2}^{T}, \gamma_{1}^{(0)^{T}}, \tilde{\gamma}_{2}^{T}\right)^{T}$, sendo $\tilde{\beta}_{2}$ e $\tilde{\gamma}_{2}$ os estimadores de máxima verossimilhança de $\beta_{2}$ e $\gamma_{2}$, respectivamente, sob a hipótese nula $H_{1}$.

A forma corrigida para a estatística escore $S_{R}$, de acordo com Cordeiro e Ferrari (1991), é dada por

$$
S_{R}^{*}=S_{R}\left[1-\frac{A_{3}}{12 u(u+2)(u+4)} S_{R}{ }^{2}-\frac{A_{2}-2 A_{3}}{12 u(u+2)} S_{R}-\frac{A_{1}-A_{2}+A_{3}}{12 u}\right],
$$

e tem distribuição $\chi_{u}^{2}$ até ordem $n^{-1}$, sob a hipótese nula $H_{1}$, onde $u=p_{1}+q_{1}$. Os coeficientes $A_{1}, A_{2}$ e $A_{3}$, são obtidos a partir das expressões (A.1) a (A.3) apresentadas no Apêndice, podendo ser escritos como

$$
\begin{aligned}
& A_{1}=3 A_{11}-6 A_{12}+6 A_{13}-6 A_{14}, \\
& A_{2}=-3 A_{21}+6 A_{22}-6 A_{23}+3 A_{24}
\end{aligned}
$$

e

$$
A_{3}=3 A_{31}+2 A_{32}
$$

Para o modelo normal em (1.1), os elementos $A_{11}, \ldots, A_{14}, A_{21}, \ldots, A_{24}, A_{31}$ e $A_{32}$ foram desenvolvidos substituindo os cumulantes apresentados no Apêndice nas expressões (A.1) a 
(A.3). Esses elementos assumem as seguintes formas matriciais

$$
\begin{aligned}
& A_{11}=1^{\prime} \Phi F Z_{2 \beta d}\left(Z_{\beta}-Z_{2 \beta}\right) Z_{2 \beta d} F \Phi 1+1^{\prime} \Phi_{1} W Z_{2 \beta d}\left(Z_{\gamma}-Z_{2 \gamma}\right) Z_{2 \beta d} W \Phi_{1} 1 \\
& +1^{\prime}\left(\Phi^{-3} \Phi_{1}^{3}-\frac{1}{2} \Phi^{-2} \Phi_{1} \Phi_{2}\right) Z_{2 \gamma d}\left(Z_{\gamma}-Z_{2 \gamma}\right) Z_{2 \beta d} W \Phi_{1} 1 \\
& +1^{\prime} \Phi_{1} W Z_{2 \beta d}\left(Z_{\gamma}-Z_{2 \gamma}\right) Z_{2 \gamma d}\left(\Phi^{-3} \Phi_{1}^{3}-\frac{1}{2} \Phi^{-2} \Phi_{1} \Phi_{2}\right) 1 \\
& +1^{\prime}\left(\Phi^{-3} \Phi_{1}^{3}-\frac{1}{2} \Phi^{-2} \Phi_{1} \Phi_{2}\right) Z_{2 \gamma d}\left(Z_{\gamma}-Z_{2 \gamma}\right) \\
& Z_{2 \gamma d}\left(\Phi^{-3} \Phi_{1}^{3}-\frac{1}{2} \Phi^{-2} \Phi_{1} \Phi_{2}\right) 1, \\
& A_{12}=1^{\prime} \Phi_{1} W Z_{2 \beta d} Z_{2 \gamma}\left(Z_{\beta d}-Z_{2 \beta d}\right) W \Phi_{1} 1 \\
& -1^{\prime} \Phi_{1} W Z_{2 \beta d} Z_{2 \gamma}\left(Z_{\gamma d}-Z_{2 \gamma d}\right) \Phi^{-3} \Phi_{1}^{3} 1 \\
& -1^{\prime}\left(\Phi^{-3} \Phi_{1}^{3}-\frac{1}{2} \Phi^{-2} \Phi_{1} \Phi_{2}\right) Z_{2 \gamma d} Z_{2 \gamma}\left(Z_{\beta d}-Z_{2 \beta d}\right) \Phi_{1} W 1 \\
& -1^{\prime}\left(\Phi^{-3} \Phi_{1}^{3}-\frac{1}{2} \Phi^{-2} \Phi_{1} \Phi_{2}\right) Z_{2 \gamma d} Z_{2 \gamma}\left(Z_{\gamma d}-Z_{2 \gamma d}\right) \Phi^{-3} \Phi_{1}^{3} 1 \\
& A_{13}=-1^{\prime} \Phi F\left\{Z_{2 \beta} \odot\left(Z_{\beta}-Z_{2 \beta}\right) \odot Z_{2 \beta}\right\} F \Phi 1 \\
& +1^{\prime} \Phi_{1} W Z_{2 \beta} \odot\left(Z_{\gamma}-Z_{2 \gamma}\right) \odot Z_{2 \beta} \Phi_{1} W 1 \\
& +1^{\prime}\left(\Phi^{-3} \Phi_{1}^{3}+\frac{1}{2} \Phi^{-2} \Phi_{1} \Phi_{2}\right) Z_{2 \gamma} \odot\left(Z_{\gamma}-Z_{2 \gamma}\right) \\
& \odot Z_{2 \gamma}\left(\Phi^{-3} \Phi_{1}^{3}-\frac{1}{2} \Phi^{-2} \Phi_{1} \Phi_{2}\right) 1 \text {, } \\
& A_{14}=\operatorname{tr}\left\{\left(3 \Phi^{-4} \Phi_{1}^{4}-\Phi_{1}^{2} \Phi_{2} \Phi^{-3}\right) Z_{2 \gamma d}\left(Z_{\gamma d}-Z_{2 \gamma d}\right)\right. \\
& +\left(2 \Phi^{-1} W \Phi_{1}^{2}-\Phi_{2} W\right) Z_{2 \gamma d}\left(Z_{\beta d}-Z_{2 \beta d}\right) \\
& \left.+2 \Phi^{-1} W \Phi_{1}^{2} Z_{2 \beta d}\left(Z_{\gamma d}-Z_{2 \gamma d}\right)\right\} \text {, } \\
& A_{21}=1^{\prime} \Phi_{1} W\left(Z_{\beta d}-Z_{2 \beta d}\right) Z_{2 \gamma}\left(Z_{\beta d}-Z_{2 \beta d}\right) \Phi_{1} W 1 \\
& +2 \times 1^{\prime} \Phi^{-3} \Phi_{1}^{3}\left(Z_{\gamma d}-Z_{2 \gamma d}\right) Z_{2 \gamma}\left(Z_{\beta d}-Z_{2 \beta d}\right) \Phi_{1} W 1 \\
& +1^{\prime} \Phi^{-3} \Phi_{1}^{3}\left(Z_{\gamma d}-Z_{2 \gamma d}\right) Z_{2 \gamma}\left(Z_{\gamma d}-Z_{2 \gamma d}\right) \Phi^{-3} \Phi_{1}^{3} 1,
\end{aligned}
$$




$$
\begin{gathered}
A_{22}=-1^{\prime} \Phi_{1} W Z_{2 \beta d}\left(Z_{\gamma}-Z_{2 \gamma}\right)\left(Z_{\beta d}-Z_{2 \beta d}\right) \Phi_{1} W 1 \\
-1^{\prime}\left(\Phi^{-3} \Phi_{1}^{3}-\frac{1}{2} \Phi^{-2} \Phi_{1} \Phi_{2}\right) Z_{2 \gamma d}\left(Z_{\gamma}-Z_{2 \gamma}\right)\left(Z_{\beta d}-Z_{2 \beta d}\right) \Phi_{1} W \quad 1 \\
-1^{\prime} \Phi_{1} W Z_{2 \beta d}\left(Z_{\gamma}-Z_{2 \gamma}\right)\left(Z_{\gamma d}-Z_{2 \gamma d}\right) \Phi^{-3} \Phi_{1}^{3} 1 \\
-1^{\prime}\left(\Phi^{-3} \Phi_{1}^{3}-\frac{1}{2} \Phi^{-2} \Phi_{1} \Phi_{2}\right) Z_{2 \gamma d}\left(Z_{\gamma}-Z_{2 \gamma}\right)\left(Z_{\gamma d}-Z_{2 \gamma d}\right) \Phi^{-3} \Phi_{1}^{3} 1 \\
A_{23}=\quad 21^{\prime} \Phi_{1} W\left(Z_{\beta}-Z_{2 \beta}\right) \odot Z_{2 \beta}\left(Z_{\gamma}-Z_{2 \gamma}\right) \Phi_{1} W 1 \\
+1^{\prime} \Phi_{1} W\left(Z_{\beta}-Z_{2 \beta}\right) \odot Z_{2 \gamma}\left(Z_{\beta}-Z_{2 \beta}\right) \Phi_{1} W 1 \\
+1^{\prime} \Phi^{-3} \Phi_{1}^{3}\left(Z_{\gamma}-Z_{2 \gamma}\right) \odot Z_{2 \gamma} \odot\left(Z_{\gamma}-Z_{2 \gamma}\right) \Phi^{-3} \Phi_{1}^{3} \\
\quad A_{24}=3 \operatorname{tr}\left\{\left(Z_{\gamma d}-Z_{2 \gamma d}\right)^{2} \Phi^{-4} \Phi_{1}^{4}\right\} \\
A_{31}= \\
\quad 1^{\prime} \Phi_{1} W\left(Z_{\beta d}-Z_{2 \beta d}\right)\left(Z_{\gamma}-Z_{2 \gamma}\right)\left(Z_{\beta d}-Z_{2 \beta d}\right) W \Phi_{1} 1 \\
+1^{\prime} \Phi^{-3} \Phi_{1}^{3}\left(Z_{\gamma d}-Z_{2 \gamma d}\right)\left(Z_{\gamma}-Z_{2 \gamma}\right)\left(Z_{\beta d}-Z_{2 \beta d}\right) W \Phi_{1} 1 \\
+1^{\prime} \Phi_{1} W\left(Z_{\beta d}-Z_{2 \beta d}\right)\left(Z_{\gamma}-Z_{2 \gamma}\right)\left(Z_{\gamma d}-Z_{2 \gamma d}\right) \Phi_{1}^{3} \Phi^{-3} 1 \\
+1^{\prime} \Phi^{-3} \Phi_{1}^{3}\left(Z_{\gamma d}-Z_{2 \gamma d}\right)\left(Z_{\gamma}-Z_{2 \gamma}\right)\left(Z_{\gamma d}-Z_{2 \gamma d}\right) \Phi_{1}^{3} \Phi^{-3} 1
\end{gathered}
$$

e

$$
\begin{aligned}
A_{32}= & 31^{\prime} \Phi_{1} W\left(Z_{\beta}-Z_{2 \beta}\right) \odot\left(Z_{\gamma}-Z_{2 \gamma}\right) \odot\left(Z_{\beta}-Z_{2 \beta}\right) \Phi_{1} W 1 \\
& +1^{\prime} \Phi^{-3} \Phi_{1}^{3}\left(Z_{\gamma}-Z_{2 \gamma}\right) \odot\left(Z_{\gamma}-Z_{2 \gamma}\right) \odot\left(Z_{\gamma}-Z_{2 \gamma}\right) \Phi^{-3} \Phi_{1}^{3} 1
\end{aligned}
$$

onde todas as matrizes envolvidas estão definidas nas Seções 2.1 e 2.2, sendo avaliadas sob o modelo restrito especificado por $H_{1}$. A notação $\odot$ indica a operação produto interno entre matrizes.

Como uma aplicação da correção tipo-Bartlett para a estatística escore, consideremos o teste da hipótese nula $H_{1}^{\prime}: \beta_{1}=0, \gamma_{1}=0$, com $p_{1}=p-1, q_{1}=q-1, x_{l p}=1$ e $s_{l q}=1$ 
para $l=1, \ldots, n$ contra a hipótese alternativa $H$ : pelo menos uma das igualdades é violada, no modelo normal definido em (1.1). A estatística escore corrigida é obtida de (3.2) e tem distribuição $\chi_{p+q-2}^{2}$ sob a hipótese nula, até ordem $n^{-1}$. Sob ${H^{\prime}}_{1}$, temos que $X=S=1$ e as matrizes $F, W, \Phi, \Phi_{1}, \Phi_{2}, Z_{2 \beta}, Z_{2 \beta d}, Z_{2 \gamma}, Z_{2 \gamma d}, Z_{\beta}, Z_{\beta d}, Z_{\gamma}, Z_{\gamma d}$ são iguais às definidas na Seção 2.2 .

Os coeficientes $A_{1}, A_{2}$ e $A_{3}$ são obtidos de (3.3) a (3.15) e dados por

$$
\begin{aligned}
A_{1}= & -\frac{3 f^{2}}{n^{2} w^{3} \phi} 1^{\prime} J 1+\frac{1}{n^{2}}\left(18 B^{2}+12 B+18\right) 1^{\prime} N 1-\frac{12}{n} p \\
& -\frac{24}{n} q+\frac{3 f^{2}}{n^{2} w^{3} \phi}+\frac{1}{n}\left(-18 B^{2}-12 B+18\right), \\
A_{2}= & -\frac{24}{n}(1+B) 1^{\prime} N N_{d} 1-\frac{12}{n}(1+B) 1^{\prime} N J_{d} 1-\frac{24}{n} 1^{\prime} J \odot N 1 \\
& -\frac{12}{n} 1^{\prime} J \odot J 1+\frac{1}{n^{2}}(156+36 B) 1^{\prime} N 1+\frac{48}{n^{2}} 1^{\prime} J 1+36 \times \operatorname{tr}\left(N_{d}^{2}\right) \\
& -\frac{6}{n} p^{2}-\frac{24}{n} q^{2}-\frac{24}{n} p q+\frac{1}{n}(48+12 B) p+\frac{24}{n}(B-1) q \\
& -\frac{138}{n}-\frac{36 B}{n}
\end{aligned}
$$

e

$$
\begin{aligned}
A_{3}= & 61^{\prime} J_{d} N J_{d} 1+24 \times 1^{\prime} N_{d} N J_{d} 1+24 \times 1^{\prime} N_{d} N N_{d} 1-\frac{72}{n} 1^{\prime} N N_{d} 1 \\
& -\frac{36}{n} 1^{\prime} N J_{d} 1+12 \times 1^{\prime} J \odot N \odot J 1+16 \times 1^{\prime} N \odot N \odot N 1 \\
& -\frac{24}{n} 1^{\prime} J \odot N 1+\frac{114}{n^{2}} 1^{\prime} N 1+\frac{24}{n^{2}} 1^{\prime} J 1-\frac{6}{n} p^{2}-\frac{24}{n} q^{2}
\end{aligned}
$$




$$
-\frac{24}{n} p q+\frac{24}{n} p+\frac{24}{n} q-\frac{82}{n}
$$

onde $B=2-\frac{\phi \delta_{2}}{\delta_{1}^{2}}$ e as matrizes $J$ e $N$ são as definidas na Seção 2.2 Considerando a função de ligação identidade para a média temos $W=I, F=0$ e as expressões de $A_{1}, A_{2}$ e $A_{3}$ em (3.16) a (3.18), respectivamente, simplificam-se muito pouco.

\subsubsection{Modelo de regressão linear simples}

Consideremos um modelo normal heterocedástico definido por (1.1) com componentes sistemáticas $d\left(\mu_{l}\right)=\eta_{l}=\beta_{2}+\beta_{1} x_{l}$ e $g\left(\phi_{l}\right)=\tau_{l}=\gamma_{2}+\gamma_{1} s_{l}$, onde $x_{l}$ e $s_{l}$ denotam os valores das variáveis explicativas $x$ e $s$, para $l=1, \ldots, n$. Para o teste de $H^{\prime}{ }_{1}: \beta_{1}=0, \gamma_{1}=0$, utilizamos a mesma notação da Seção 2.2.1, encontrando, a partir de (3.16) a (3.18) as seguintes expressões $A_{1}, A_{2}$ e $A_{3}$

$$
\begin{gathered}
A_{1}=-\frac{36}{n} \\
A_{2}=-\frac{114}{n}-\frac{24}{n} \frac{v_{x, s}^{2}}{v_{2 x}, v_{2 s}}+\frac{36}{n} \frac{v_{4 s}}{v_{2 s}^{2}}
\end{gathered}
$$

e

$$
A_{3}=\frac{18}{n} \frac{v_{2 x, s}^{2}}{v_{2 x}^{2} v_{2 s}}+\frac{8}{n} \frac{v_{2 x, s} v_{3 s}}{v_{2 x} v_{2 s}^{2}}+\frac{40}{n} \frac{v_{3 s}^{2}}{v_{2 s}^{3}} .
$$

É interessante observar que os termos $A_{1}, A_{2}$ e $A_{3}$ em (3.19) a (3.21) não dependem das funções de ligação consideradas para a média e para o parâmetro de precisão.

\subsection{Testes sobre o parâmetro de precisão}

Consideremos o modelo normal definido em (1.1) e a partição $\gamma^{T}=\left(\gamma_{1}{ }^{T}, \gamma_{2}{ }^{T}\right)$ para o vetor $\gamma$. onde $\gamma_{1}=\left(\gamma_{1}, \ldots, \gamma_{q_{1}}\right)^{T}$ e $\gamma_{2}=\left(\gamma_{q_{1}+1}, \ldots, \gamma_{q}\right)^{T}$, com $q_{1} \leq q$. Essa decomposição induz a 
correspondente partição $S=\left(S_{1}, S_{2}\right)$.

Estamos interessados em testar a hipótese $H_{2}: \gamma_{1}=\gamma_{1}{ }^{(0)}$ contra $H: \gamma_{1} \neq \gamma_{1}{ }^{(0)}$, onde $\gamma_{1}{ }^{(0)}$ é um vetor conhecido de dimensão $q_{1}$.

Seja $\theta_{1}=\gamma_{1}$ o vetor de parâmetros de interesse e $\theta_{2}=\left(\gamma_{2}^{T}, \beta^{T}\right)^{T}$ o vetor de parâmetros de perturbação. A função escore correspondente é dada por

$$
U=\left(U_{1}^{T}, U_{2}^{T}\right)^{T}
$$

onde

$$
U_{1}=\partial \ell(\beta, \gamma) / \partial \theta_{1}=U_{\gamma_{1}}\left(\beta, \gamma_{1}, \gamma_{2}\right)
$$

$\mathrm{e}$

$$
U_{2}^{T}=\partial \ell(\beta, \gamma) / \partial \theta_{2}=\left(U_{\gamma_{2}}^{T}\left(\beta, \gamma_{1}, \gamma_{2}\right), U^{T}\left(\beta, \gamma_{1}, \gamma_{2}\right)\right)^{T}
$$

Além disto, a matriz de informação total de Fisher correspondente é dada por

$$
K=\left(\begin{array}{ll}
K_{11} & K_{12} \\
K_{21} & K_{22}
\end{array}\right)=\left(\begin{array}{ccc}
K_{\gamma_{11}} & K_{\gamma_{12}} & 0 \\
K_{\gamma_{21}} & K_{\gamma_{22}} & 0 \\
0 & 0 & K_{\beta, \beta}
\end{array}\right),
$$

onde as matrizes $K_{\gamma_{11}}, K_{\gamma_{12}}=K_{\gamma_{21}}^{T}, K_{\gamma_{22}}$ e $K_{\beta, \beta}$ estão definidas na Seção 2.2. Temos

$$
K^{-1}=\left(\begin{array}{ccc}
K_{\gamma}^{11} & K_{\gamma}^{12} & 0 \\
K_{\gamma}^{21} & K_{\gamma}^{22} & 0 \\
0 & 0 & K_{\beta, \beta}^{-1}
\end{array}\right)
$$

A estatística escore $S_{R}$ para o teste de $H_{2}$ tem a forma

$$
S_{R}=\tilde{U}_{1}^{T} \tilde{K}_{\gamma}^{11} \tilde{U}_{1}
$$

onde $U_{1}$ e $K^{11}$ estão avaliadas em $\left(\tilde{\beta}^{T}, \gamma_{1}^{(0) T}, \tilde{\gamma}_{2}^{T}\right)^{T}$, sendo $\tilde{\beta}$ e $\tilde{\gamma}_{2}$ os estimadores de máxima verossimilhança de $\beta$ e $\gamma_{2}$ sob $H_{2}$. 
A forma corrigida para a estatística escore $S_{R}$, de acordo com Cordeiro e Ferrari (1991), é dada por

$$
S_{R}{ }^{*}=S_{R}\left[1-\frac{A_{3}}{12 q_{1}\left(q_{1}+2\right)\left(q_{1}+4\right)} S_{R}{ }^{2}-\frac{A_{2}-2 A_{3}}{12 q_{1}\left(q_{1}+2\right)} S_{R}-\frac{A_{1}-A_{2}+A_{3}}{12 q_{1}}\right],
$$

e tem distribuição $\chi_{q_{1}}^{2}$ até ordem $O\left(n^{-1}\right)$ sob a hipótese nula $H_{2}$. As quantidades em (3.3) a (3.5) para obter os coeficientes $A_{1}, A_{2}$ e $A_{3}$, são obtidas a partir das expressões (3.6) a (3.15) considerando que $Z_{\beta}=Z_{2 \beta}$ e são dadas por

$$
\begin{aligned}
& A_{11}=1^{\prime} \Phi_{1} W Z_{\beta d}\left(Z_{\gamma}-Z_{2 \gamma}\right) Z_{\beta d} W \Phi_{1} 1+21^{\prime}\left(\Phi^{-3} \Phi_{1}^{3}-\frac{1}{2} \Phi^{-2} \Phi_{1} \Phi_{2}\right) Z_{2 \gamma d} \\
& \left(Z_{\gamma}-Z_{2 \gamma}\right) Z_{\beta d} W \Phi_{1} 1+1^{\prime}\left(\Phi^{-3} \Phi_{1}^{3}-\frac{1}{2} \Phi^{-2} \Phi_{1} \Phi_{2}\right) Z_{2 \gamma d}\left(Z_{\gamma}-Z_{2 \gamma}\right) \\
& Z_{2 \gamma d}\left(\Phi^{-3} \Phi_{1}^{3}-\frac{1}{2} \Phi^{-2} \Phi_{1} \Phi_{2}\right) 1, \\
& A_{12}=-1^{\prime} \Phi_{1} W Z_{\beta d} Z_{2 \gamma}\left(Z_{\gamma d}-Z_{2 \gamma d}\right) \Phi^{-3} \Phi_{1}^{3} 1 \\
& -1^{\prime}\left(\Phi^{-3} \Phi_{1}^{3}-\frac{1}{2} \Phi^{-2} \Phi_{1} \Phi_{2}\right) Z_{2 \gamma d} Z_{2 \gamma}\left(Z_{\gamma d}-Z_{2 \gamma d}\right) \Phi^{-3} \Phi_{1}^{3} 1 \\
& A_{13}=1^{\prime} \Phi_{1} W Z_{\beta} \odot\left(Z_{\gamma}-Z_{2 \gamma}\right) \odot Z_{\beta} \Phi_{1} W 1+1^{\prime}\left(\Phi^{-3} \Phi_{1}^{3}-\frac{1}{2} \Phi^{-2} \Phi_{1} \Phi_{2}\right) Z_{2 \gamma} \\
& \odot\left(Z_{\gamma}-Z_{2 \gamma}\right) \odot Z_{2 \gamma}\left(\Phi^{-3} \Phi_{1}^{3}-\frac{1}{2} \Phi^{-2} \Phi_{1} \Phi_{2}\right) 1 \\
& A_{14}=\operatorname{tr}\left\{\left(3 \Phi_{1}^{4} \Phi^{-4}-\Phi_{1}^{2} \Phi_{2} \Phi^{-3}\right) Z_{2 \gamma d}\left(Z_{\gamma d}-Z_{2 \gamma d}\right)\right. \\
& \left.+2 \Phi^{-1} W \Phi_{1}^{2} Z_{\beta d}\left(Z_{\gamma d}-Z_{2 \gamma d}\right)\right\} \\
& A_{21}=1^{\prime} \Phi^{-3} \Phi_{1}^{3}\left(Z_{\gamma d}-Z_{2 \gamma d}\right) Z_{2 \gamma}\left(Z_{\gamma d}-Z_{2 \gamma d}\right) \Phi^{-3} \Phi_{1}^{3} 1, \\
& A_{22}=-1^{\prime} \Phi_{1} W Z_{\beta d}\left(Z_{\gamma}-Z_{2 \gamma}\right)\left(Z_{\gamma d}-Z_{2 \gamma d}\right) \Phi^{-3} \Phi_{1}^{3} 1
\end{aligned}
$$




$$
-1^{\prime}\left(\Phi^{-3} \Phi_{1}^{3}-\frac{1}{2} \Phi^{-2} \Phi_{1} \Phi_{2}\right) Z_{2 \gamma d}\left(Z_{\gamma}-Z_{2 \gamma}\right)\left(Z_{\gamma d}-Z_{2 \gamma d}\right) \Phi^{-3} \Phi_{1}^{3} 1
$$

$$
\begin{gathered}
A_{23}=1^{\prime} \Phi^{-3} \Phi_{1}^{3}\left(Z_{\gamma}-Z_{2 \gamma}\right) \odot Z_{2 \gamma} \odot\left(Z_{\gamma}-Z_{2 \gamma}\right) \Phi^{-3} \Phi_{1}^{3} 1 \\
A_{24}=3 \operatorname{tr}\left\{\left(Z_{\gamma d}-Z_{2 \gamma d}\right)^{2} \Phi_{1}{ }^{4} \Phi^{-4}\right\}
\end{gathered}
$$

$$
A_{31}=1^{\prime} \Phi^{-3} \Phi_{1}^{3}\left(Z_{\gamma d}-Z_{2 \gamma d}\right)\left(Z_{\gamma}-Z_{2 \gamma}\right)\left(Z_{\gamma d}-Z_{2 \gamma d}\right) \Phi^{-3} \Phi_{1}^{3} 1
$$

e

$$
A_{32}=1^{\prime} \Phi^{-3} \Phi_{1}^{3}\left(Z_{\gamma}-Z_{2 \gamma}\right) \odot\left(Z_{\gamma}-Z_{2 \gamma}\right) \odot\left(Z_{\gamma}-Z_{2 \gamma}\right) \Phi^{-3} \Phi_{1}^{3} 1
$$

Como uma aplicação vamos considerar o modelo normal dado em (1.1) com função de ligação identidade para a média, função de ligação qualquer para o parâmetro de precisão e o teste da hipótese $H_{2}^{\prime}: \gamma_{1}=0, \operatorname{com} q_{1}=q-1$ e $s_{l q}=1$ para $l=1, \ldots, n$ contra $H: \gamma_{1} \neq 0$. Aqui, a estatística escore corrigida, dada em (3.1), para testar $H_{2}$, tem distribuição $\chi_{q_{1}}^{2}$, onde $q_{1}=q-1$. Então, sob $H_{2}$ temos que $Z_{2 \gamma}=2 \phi^{2}\left(n \delta_{1}^{2}\right)^{-1} 11^{T}, Z_{2 \gamma d}=2 \phi^{2}\left(n \delta_{1}^{2}\right)^{-1} I$, $Z_{\gamma}=\left(2 \phi^{2} / \delta_{1}^{2}\right) N, Z_{\gamma d}=\left(2 \phi^{2} / \delta_{1}^{2}\right) N_{d}, Z_{\beta}=(1 / \phi) J$ e $Z_{\beta d}=(1 / \phi) J_{d}$, onde as matrizes $J, N$ e as quantidades $\delta_{1}$ e $\delta_{2}$ estão definidas na Seção 2.2. Assim os termos em $A_{1}, A_{2}$ e $A_{3}$ vêm de (3.3) a (3.5) e de (3.23) a (3.32) e $A_{1}-A_{3}$ são dados por:

$$
\begin{aligned}
A_{1}= & 61^{\prime} J_{d} N J_{d} 1+121^{\prime} J \odot N \odot J 1-24 \operatorname{tr}\left(J_{d} N_{d}\right)+\frac{12}{n} B \\
& \left(1^{\prime} N J_{d} 1-1\right)+\frac{18}{n}\left(2-\frac{\phi \delta_{2}}{\delta_{1}^{2}}\right)^{2}\left(1^{\prime} N 1-1\right)-\frac{6 p}{n}(p+2) \\
& +\frac{24 q}{n}(p-1)+\frac{24}{n},
\end{aligned}
$$




$$
\begin{gathered}
A_{2}=36 \operatorname{tr}\left(N_{d}^{2}\right)-241^{\prime} J_{d} N N_{d} 1+\frac{24}{n} 1^{\prime} J_{d} N 1-\frac{24}{n} B 1^{\prime} N N_{d} 1 \\
+\frac{24}{n^{2}}\left(6-\frac{\phi \delta_{2}}{\delta_{1}^{2}}\right) 1^{\prime} N 1-\frac{24 q}{n}\left(q+1+\frac{\phi \delta_{2}}{\delta_{1}^{2}}\right)-\frac{12}{n}\left(7-2 \frac{\phi \delta_{2}}{\delta_{1}^{2}}\right), \\
A_{3}=241^{\prime} N_{d} N N_{d} 1-\frac{48}{n} 1^{\prime} N_{d} N 1+161^{\prime} N \odot N \odot N 1 \\
\quad+\frac{72}{n^{2}} 1^{\prime} N 1-\frac{8}{n}\left(3 q^{2}+5\right) .
\end{gathered}
$$

\subsubsection{Modelo de regressão linear simples}

Consideremos o modelo normal em (1.1) com $d\left(\mu_{l}\right)=\eta_{l}=\beta_{2}+\beta_{1} x_{l}$ e $g\left(\phi_{l}\right)=\tau_{l}=\gamma_{2}+\gamma_{1} s_{l}$, onde $x_{l}$ e $s_{l}$ denotam os valores das variáveis explicativas $x$ e $s$, para $l=1, \ldots, n$. Utilizando a notação da Seção 2.2.1, temos que a estatística escore corrigida para testar a hipótese $H^{\prime}{ }_{2}: \gamma_{1}=0$ é obtida de (3.22) com seus coeficientes $A_{1}, A_{2}$ e $A_{3}$ obtidos de (3.3) a (3.5) e (3.23) a (3.32). Encontramos

$$
\begin{aligned}
& A_{1}= 3 \delta_{1}^{2} 1^{T} W Z_{\beta d} Z_{\gamma} Z_{\beta d} W 1-\frac{6 \phi^{2}}{n}\left\{\operatorname{tr}\left(W Z_{\beta d}\right)\right\}^{2} \\
&+ \frac{6}{n}\left(\frac{2 \delta_{1}^{2}}{\phi}-\delta_{2}\right) 1^{\prime} Z_{\gamma} Z_{\beta d} W 1+6 \delta_{1}^{2} 1^{T} W Z_{\beta d} \odot Z_{\gamma} \odot Z_{\beta} W 1 \\
&- \frac{12 \phi^{2}}{n} 1^{T} W Z_{\beta} \odot Z_{\beta} W 1-\frac{12 \phi^{2}}{n} \operatorname{tr}\left(W Z_{\beta d} Z_{\gamma d}\right)-\frac{24}{n} \\
& A_{2}=-\frac{6 \delta_{1}^{4}}{\phi^{3}} 1^{T} W Z_{\beta d} Z_{\gamma} Z_{\gamma d} 1+\frac{12 \delta_{1}^{2}}{n \phi} 1^{T} W Z_{\beta d} Z_{\gamma} 1 \\
& \frac{24 \phi}{n} 1^{T} W Z_{\beta d} 1+\frac{36}{n}\left(\gamma_{2 s}+3\right)+\frac{36}{n} \gamma_{2 s}-\frac{36}{n}
\end{aligned}
$$




$$
A_{3}=\frac{40 v_{3 s}^{2}}{n v_{2 s}^{3}}=\frac{40}{n} \gamma_{1 s}^{2},
$$

onde $\gamma_{1 s}=\frac{v_{3 s}}{v_{2 s}^{3 / 2}}$ e $\gamma_{2 s}=\frac{v_{4 s}}{v_{2 s}^{2}}$ são respectivamente as medidas de assimetria e curtose padronizadas de $s$ É interessante observar que, neste caso, as expressões $A_{1}$ e $A_{2}$ dependem das funções de ligação para a média e para o parâmetro de precisão. Se, neste caso, considerarmos uma função ligação identidade para a média e centrarmos a variável explicativa $s$, as expressões (3.33) a (3.35) igualam-se às expressões (6) a (8) de Cribari-Neto e Ferrari (1995a).

Considerando o modelo normal com ligação identidade para a média e logaritmica para o parâmetro de precisão, obtemos

$$
\begin{aligned}
& A_{1}=6\left(\frac{4 v_{x, s}^{2}}{n v_{2 x} v_{2 s}}-\frac{4 v_{2 x, 2 s}}{n v_{2 x} v_{2 s}}+\frac{3 v_{2 x, s}^{2}}{n v_{2 x}^{2} v_{2 s}}\right), \\
& A_{2}=-12\left(\frac{6}{n}-\frac{3}{n} \frac{v_{4 s}}{v_{2 s}^{2}}+\frac{2}{n} \frac{v_{2 x, s} v_{3 s}}{v_{2 s}^{2} v_{2 x}}\right) .
\end{aligned}
$$

sendo que $A_{3}$ é o mesmo da expressão (3.35). Para $X=S$, temos $A_{1}=-\frac{6}{n}\left(8+4 \gamma_{2 s}-3 \gamma_{1 s}^{2}\right)$ e $A_{2}=\frac{12}{n}\left(3+3 \gamma_{2 s}-2 \gamma_{1 s}^{2}\right)$ que igualam-se às expressões de (9) e (10) de Cribari-Neto e Ferrari.

\subsection{Testes sobre a média}

Consideremos o modelo normal em (1.1) e a partição $\beta^{T}=\left(\beta_{1}{ }^{T}, \beta_{2}{ }^{T}\right)$ para o vetor $\beta$, onde $\beta_{1}=\left(\beta_{1}, \ldots, \beta_{p_{1}}\right)^{T}$ e $\beta_{2}=\left(\beta_{p_{1}+1}, \ldots, \beta_{p}\right)^{T}$, com $p_{1} \leq p$. Essa decomposição induz a correspondente partição $X=\left(X_{1}, X_{2}\right)$. 
Estamos interessados em testar a hipótese $H_{3}: \beta_{1}=3_{1}{ }^{(0)}$ contra $H: \beta_{1} \neq \beta_{1}{ }^{\left({ }^{0}\right)}$, onde $\beta_{1}{ }^{(0)}$ é um vetor conhecido de dimensão $p_{1}$.

Seja $\theta_{1}=\beta_{1}$ o vetor de parâmetros de interesse e $\theta_{2}=\left(\beta_{2}^{T}, \gamma^{T}\right)^{T}$ o vetor de parâmetros de perturbação. A função escore correspondente é dada por

$$
U=\left(U_{1}^{T}, U_{2}^{T}\right)^{T}
$$

onde

$$
U_{1}=\partial \ell(\beta, \gamma) / \partial \theta_{1}=U_{\beta_{1}}\left(\beta_{1}, 3_{2}, \gamma\right)
$$

e

$$
U_{2}^{T}=\partial \ell(\beta, \gamma) / \partial \theta_{2}=\left(U_{\beta_{2}}^{T}\left(\beta_{1}, \beta_{2}, \gamma\right), \quad L_{\gamma}^{-T}\left(\beta_{1}, \beta_{2}, \gamma\right)\right)^{T}
$$

A matriz de informação total de Fisher correspondente é dada por

$$
K=\left(\begin{array}{ll}
K_{11} & K_{12} \\
K_{21} & K_{22}
\end{array}\right)=\left(\begin{array}{ccc}
K_{\beta_{11}} & K_{\beta_{12}} & 0 \\
K_{\beta_{21}} & K_{\beta_{22}} & 0 \\
0 & 0 & K_{\gamma, \gamma}
\end{array}\right)
$$

onde as matrizes $K_{\beta_{11}}, K_{\beta_{12}}^{T}=K_{\beta_{21}}, K_{\beta_{22}}$ e $K_{\gamma \gamma}$ estão definidas na Seção 2.2. Temos

$$
K^{-1}=\left(\begin{array}{ccc}
K_{\beta}^{11} & K_{\beta}^{12} & 0 \\
K_{\beta}^{21} & K_{\beta}^{22} & 0 \\
0 & 0 & K_{\gamma, \gamma}^{-1}
\end{array}\right)
$$

A estatística escore $S_{R}$, para o teste de $H_{3}$ assume a expressão

$$
S_{R}=\tilde{U}_{1}^{T} \tilde{K}_{\beta}^{11} \tilde{U}_{1}
$$

onde $\tilde{U}_{1}$ e $\tilde{K}^{11}$ estão avaliadas em $\left(\beta_{1}^{(0) T}, \tilde{\beta}_{2}^{T}, \tilde{\gamma}^{T}\right)^{T}$, sendo $\tilde{3}_{2}^{T}$ e $\tilde{\gamma}$ os estimadores de máxima verossimilhança de $\beta_{2}$ e $\gamma$ sob $H_{3}$. 
A forma corrigida para a estatística escore $S_{R}$, de acordo com Cordeiro e Ferrari (1991), é dada pela expressão

$$
S_{R}{ }^{*}=S_{R}\left[1-\frac{A_{3}}{12 p_{1}\left(p_{1}+2\right)\left(p_{1}+4\right)} S_{R}{ }^{2}-\frac{A_{2}-2 A_{3}}{12 p_{1}\left(p_{1}+2\right)} S_{R}-\frac{A_{1}-A_{2}+A_{3}}{12 p_{1}}\right],
$$

e tem distribuição $\chi_{p_{1}}^{2}$ até ordem $O\left(n^{-1}\right)$ sob a hipótese nula $H_{3}$. As quantidades em (3.3) a (3.5) para obter os coeficientes $A_{1}, A_{2}$ e $A_{3}$, são obtidas a partir das expressões (3.6) a (3.15) considerando que $Z_{\gamma}=Z_{2 \gamma}$ e são dadas por

$$
\begin{aligned}
& A_{11}=1^{\prime} \Phi F Z_{2 \beta d}\left(Z_{\beta}-Z_{2 \beta}\right) Z_{2 \beta d} F \Phi 1, \\
& A_{12}=1^{\prime}\left\{\Phi_{1} W Z_{2 \beta d}-\left(\Phi^{-3} \Phi_{1}^{3}-\frac{1}{2} \Phi^{-2} \Phi_{1} \Phi_{2}\right) Z_{\gamma d}\right\} Z_{\gamma} \\
& \left(Z_{\beta d}-Z_{2 \beta d}\right) \Phi_{1} W 1 \text {, } \\
& A_{13}=-1^{\prime} \Phi F\left\{Z_{2 \beta} \odot\left(Z_{\beta}-Z_{2 \beta}\right) \odot Z_{2 \beta}\right\} F \Phi 1, \\
& A_{14}=\operatorname{tr}\left\{\left(2 \Phi^{-1} W \Phi_{1}^{2}-\Phi_{2} W\right) Z_{\gamma d}\left(Z_{\beta d}-Z_{2 \beta d}\right)\right\}, \\
& A_{21}=1^{\prime} \Phi_{1} W\left(Z_{\beta d}-Z_{2 \beta d}\right) Z_{\gamma}\left(Z_{\beta d}-Z_{2 \beta d}\right) \Phi_{1} W 1, \\
& A_{22}=A_{24}=0 \\
& A_{23}=1^{\prime}\left\{\Phi_{1} W\left(Z_{\beta}-Z_{2 \beta}\right) \odot Z_{\gamma} \odot\left(Z_{\beta}-Z_{2 \beta}\right) \Phi_{1} W 1\right.
\end{aligned}
$$

e

$$
A_{31}=A_{32}=0 \text {. }
$$


Como uma aplicação consideremos o modelo normal em (1.1) com funções de ligação quaisquer para a média $(d(\mu)=\eta=X \beta)$ e para o parâmetro de precisão $(g(\phi)=\tau=S \gamma)$, $S=1$ e a hipótese $H_{3}^{\prime}: \beta_{1}=0 \operatorname{com} p_{1}=p-1$ e $x_{l p}=1$, para $l=1, \ldots, n$. A estatística escore corrigida, dada em (3.36). tem distribuição $\chi_{p_{1}}^{2}$ com erro de ordem $O\left(n^{-2}\right)$. Os termos em $A_{1}, A_{2}$ e $A_{3}$ vêm de (3.3) a (3.5) e de (3.37) a (3.44) sendo que $A_{1}, A_{2}$ e $A_{3}$ resultam em

$$
\begin{gathered}
A_{1}=-\frac{3}{n}\left\{\frac{f^{2}}{w^{3} \phi}\left(\frac{1}{n} 1^{\prime} J 1-1\right)+4(p-1)\right\} \\
A_{2}=\frac{6}{n}\left(\frac{4}{n} 1^{\prime} J 1-p^{2}-3\right)
\end{gathered}
$$

e $A_{3}=0$.

Podemos observar que, neste caso, $A_{1}$ depende apenas da função de ligação considerada para a média, enquanto que $A_{2}$ não depende das funções de ligações adotadas para a média e para o parâmetro de precisão. 


\section{Capítulo 4}

\section{Resultados de simulações e aplicação a dados reais}

\subsection{Resultados de simulações}

Apresentamos, nesta seção, um estudo de simulação onde comparamos os desempenhos das estatísticas da razão de verossimilhança $(L R)$ e escore $\left(S_{R}\right)$, não corrigidas, e de suas versões aperfeiçoadas por fatores de correção de Bartlett e tipo-Bartlett, respectivamente, associadas a três testes de hipóteses para os modelos normais heterocedásticos definidos em (1.1). Para este estudo, consideramos um modelo com

$$
\mu_{l}=\beta_{2}+\beta_{1} x_{l}
$$

e

$$
-\log \left(\phi_{l}\right)=\gamma_{2}+\gamma_{1} x_{l}
$$

para $l=1, \ldots, n$. Os valores da variável independente $x$ foram obtidos de maneira a estabilizar os coeficientes de assimetria e curtose para diferentes tamanhos de amostras. Para 
$n=10$, esses valores foram tomados das distribuições $\mathrm{U}(0,1)\left(\gamma_{1 x}^{2}=0.00, \gamma_{2 x}=-1,22\right)$, $N(0,1)\left(\gamma_{1 x}^{2}=0,00, \gamma_{2 x}=-0,75\right)$ e $\operatorname{LN}\left(0,1.25^{2}\right)\left(\gamma_{1 x}^{2}=3,21, \gamma_{2 x}=2,17\right)$ através da expressão

$$
x_{l}=\text { quantil }\left(\frac{l-0.5}{10}\right), l=1, \ldots, 10 .
$$

Para outros tamanhos de amostras, os valores de $x_{l}$ foram replicados a partir dos obtidos para $n=10$. Para $n=20$, por exemplo, temos $x_{1}, x_{1}, x_{2}, x_{2}, \ldots, x_{10}, x_{10}$, onde $x_{1}, \ldots, x_{10}$ são os dez valores gerados das distribuições mencionadas. De forma análoga, obtivemos os valores de $x_{l}$ para outros tamanhos de amostras.

Foram geradas 10.000 amostras de $n$ observações $(n=10,20,30,40)$ de um modelo normal heterocedástico através do pacote computacional S-Plus (versão 4.5) considerando $\beta_{1}=0, \beta_{2}=1, \gamma_{1}=0$ e $\gamma_{2}=1$.

Para cada amostra os parâmetros foram estimados sob as hipóteses nulas $H^{\prime}{ }_{1}: \beta_{1}=\gamma_{1}=$ $0, H_{2}^{\prime}: \gamma_{1}=0$ e $H^{\prime}{ }_{3}: \beta_{1}=0$ e sob o modelo irrestrito, utilizando o processo iterativo escore de Fisher, discutido na Seção 1.2 do Capítulo 1, por meio da linguagem de programação Fortran (versão 77). Com base nos resultados das Seções 2.2.1, 2.3.1, 2.4, 3.2.1, 3.3.1 e 3.4, calculamos, em seguida, os valores das estatísticas $L R, S_{R}, L R^{*}$ e $S_{R}^{*}$ e suas respectivas taxas de rejeição, isto é, as proporções de vezes em que $L R, L R^{*}, S_{R}$ e $S_{R}^{*}$ excederam os valores críticos $x_{\alpha}$ obtidos da distribuição $\chi_{q}^{2}$ para os níveis nominais $\alpha=1,0 ; 2,5 ; 5,0$ e $10,0 \%$. Para as hipóteses $\mathrm{H}^{\prime}{ }_{1}, \mathrm{H}^{\prime}{ }_{2}$ e $H^{\prime}{ }_{3}$ consideradas, os valores de $q$ são, respectivamente, 2,1 e 1 . As taxas de rejeição estão exibidas nas Tabelas 4.1 a 4.9. Analisando essas tabelas, podemos observar que

- As taxas de rejeição de $L R$ estão, em geral, mais próximas dos correspondentes níveis nominais, para os testes das três hipóteses, quando a variável independente é obtida da distribuição uniforme. Para a estatística $S_{R}$, as taxas de rejeição estão mais próximas dos correspondentes níveis nominais quando a variável independente é obtida da dis- 
tribuição uniforme no caso do teste da hipótese ${H^{\prime}}_{2}$ e da distribuição normal para os testes das outras hipóteses. Em resumo, as taxas de rejeição de $L R$ e de $S_{R}$ estão mais próximas dos correspondentes níveis nominais quando a variável independente é obtida de uma distribuição simétrica com coeficiente de curtose pequeno ou moderado.

- As taxas de rejeição de $S_{R}$ e de $S_{R}^{*}$ estão, em geral, mais próximas dos correspondentes níveis nominais do que as de $L R$ e de $L R^{*}$.

- Na maioria dos casos, as taxas de rejeição de $L R^{*}$ e de $S_{R}^{*}$ estão mais próximas dos níveis nominais do que as correspondentes taxas para $L R$ e $S_{R}$.

- À medida que o tamanho da amostra cresce, as taxas de rejeição de $L R, S_{R}, L R^{*}$ e $S_{R}^{*}$ se aproximam dos correspondentes níveis nominais.

- As taxas de rejeição de $L R$ e de $L R^{*}$ são, em geral, maiores do que os correspondentes níveis nominais, enquanto que as taxas de rejeição de $S_{R}$ e de $S_{R}^{*}$ são menores, indicando então que o teste escore e sua versão corrigida são mais conservativos do que o teste da razão de verossimilhança e sua versão corrigida. Em testes com restrições lineares nos parâmetros da regressão em certos modelos normais lineares, Breusch (1979) mostrou que é válida a relação $L R \geq S_{R}$ para quaisquer conjuntos de dados (vide também Evans e Savin, 1982). Em outras palavras, estes autores já haviam notado que o teste escore é mais conservativo que o da razão de verossimilhança. 
Tabela 4.1: Taxas de rejeição para o teste da hipótese $H_{1}^{\prime}: \beta_{1}=\gamma_{1}=0$.

Valores da variável independente obtidos da $\mathrm{U}(0,1)$.

\begin{tabular}{rrrrrrrrrrrr}
\hline$n$ & $\alpha(\%)$ & $L R$ & $L R^{*}$ & $S_{R}$ & $S_{R}^{*}$ & $n$ & $\alpha(\%)$ & $L R$ & $L R^{*}$ & $S_{R}$ & $S_{R}^{*}$ \\
\hline \multirow{4}{*}{10} & 10 & 20.1 & 12.3 & 8.0 & 9.6 & & 10 & 12.8 & 10.4 & 9.5 & 10.1 \\
& 5 & 12.3 & 6.8 & 2.9 & 4.5 & 30 & 5 & 6.8 & 5.1 & 4.2 & 4.7 \\
& 2.5 & 7.6 & 3.8 & 0.8 & 2.0 & & 2.5 & 3.5 & 2.5 & 2.1 & 2.4 \\
& 1 & 4.4 & 1.8 & 0.1 & 0.5 & & 1 & 1.5 & 1.0 & 0.6 & 0.9 \\
\hline \multirow{3}{*}{20} & 10 & 13.6 & 10.2 & 8.8 & 9.6 & & 10 & 12.0 & 10.2 & 9.9 & 10.2 \\
& 5 & 7.5 & 5.1 & 4.1 & 4.9 & 40 & 5 & 6.4 & 5.1 & 4.6 & 5.1 \\
& 2.5 & 3.9 & 2.5 & 1.7 & 2.4 & & 2.5 & 3.4 & 2.6 & 2.2 & 2.6 \\
& 1 & 1.8 & 1.0 & 0.5 & 0.9 & & 1.0 & 1.5 & 1.1 & 0.8 & 1.0 \\
\hline
\end{tabular}

Tabela 4.2: Taxas de rejeição para o teste da hipótese $H_{2}^{\prime}: \gamma_{1}=0$.

Valores da variável independente obtidos da $U(0,1)$.

\begin{tabular}{rrrrrrrrrrrr}
\hline$n$ & $\alpha(\%)$ & $L R$ & $L R^{*}$ & $S_{R}$ & $S_{R}^{*}$ & $n$ & $\alpha(\%)$ & $L R$ & $L R^{*}$ & $S_{R}$ & $S_{R}^{*}$ \\
\hline & 10 & 18.8 & 13.2 & 6.3 & 8.3 & & 10 & 12.6 & 10.3 & 9.2 & 10.0 \\
10 & 5 & 11.8 & 7.3 & 2.3 & 3.8 & 30 & 5 & 6.6 & 5.3 & 4.2 & 4.9 \\
& 2.5 & 7.6 & 4.1 & 0.9 & 1.7 & & 2.5 & 3.4 & 2.6 & 2.0 & 2.4 \\
& 1 & 4.3 & 2.1 & 0.2 & 0.6 & & 1 & 1.4 & 1.0 & 0.7 & 0.9 \\
\hline & 10 & 13.4 & 10.4 & 8.3 & 9.5 & & 10 & 11.5 & 10.0 & 9.4 & 10.1 \\
20 & 5 & 7.3 & 5.3 & 3.9 & 4.9 & 40 & 5 & 6.2 & 5.3 & 4.8 & 5.3 \\
& 2.5 & 4.0 & 2.6 & 1.5 & 2.3 & & 2.5 & 3.5 & 2.8 & 2.4 & 2.8 \\
& 1 & 2.0 & 1.3 & 0.6 & 0.8 & & 1 & 1.5 & 1.1 & 0.7 & 0.9 \\
\hline
\end{tabular}


Tabela 4.3: Taxas de rejeição para o teste da hipótese $H^{\prime}{ }_{3}: \beta_{1}=0$.

Valores da variável independente obtidos da $\mathrm{U}(0,1)$.

\begin{tabular}{rrrrrrrrrrrr}
\hline$n$ & $\alpha(\%)$ & $L R$ & $L R^{*}$ & $S_{R}$ & $S_{R}^{*}$ & $n$ & $\alpha(\%)$ & $L R$ & $L R^{*}$ & $S_{R}$ & $S_{R}^{*}$ \\
\hline & 10 & 17.7 & 12.6 & 9.1 & 9.8 & & 10 & 12.1 & 10.2 & 10.2 & 9.9 \\
10 & 5 & 12.0 & 8.6 & 6.1 & 6.1 & 30 & 5 & 6.6 & 5.1 & 4.7 & 4.8 \\
& 2.5 & 9.0 & 6.9 & 5.1 & 5.1 & & 2.5 & 3.6 & 2.5 & 2.2 & 2.4 \\
& 1 & 7.1 & 5.7 & 4.7 & 4.6 & & 1 & 1.5 & 1.0 & 0.7 & 0.8 \\
\hline \multirow{2}{*}{20} & 10 & 12.5 & 10.0 & 9.2 & 9.4 & & 10 & 11.5 & 10.0 & 9.8 & 9.9 \\
& 5 & 7.3 & 5.3 & 4.5 & 4.6 & 40 & 5 & 5.9 & 5.0 & 4.9 & 4.9 \\
& 2.5 & 4.1 & 2.9 & 2.2 & 2.4 & & 2.5 & 3.1 & 2.3 & 2.1 & 2.3 \\
& 1 & 2.4 & 1.6 & 1.4 & 1.2 & & 1 & 1.3 & 1.0 & 0.8 & 0.9 \\
\hline
\end{tabular}

Tabela 4.4: Taxas de rejeição para o teste da hipótese $H_{1}^{\prime}: \beta_{1}=\gamma_{1}=0$.

Valores da variável independente obtidos da $\mathrm{N}(0,1)$.

\begin{tabular}{rrrrrrrrrrrr}
\hline$n$ & $\alpha(\%)$ & $L R$ & $L R^{*}$ & $S_{R}$ & $S_{R}^{*}$ & $n$ & $\alpha(\%)$ & $L R$ & $L R^{*}$ & $S_{R}$ & $S_{R}^{*}$ \\
\hline \multirow{2}{*}{10} & 10 & 21.6 & 13.6 & 8.6 & 9.8 & & 10 & 12.8 & 10.3 & 9.5 & 10.0 \\
& 5 & 13.7 & 7.6 & 3.7 & 4.5 & 30 & 5 & 6.9 & 5.2 & 4.6 & 4.8 \\
& 2.5 & 8.9 & 4.2 & 1.3 & 1.8 & & 2.5 & 3.7 & 2.6 & 2.2 & 2.4 \\
& 1 & 4.9 & 2.0 & 0.3 & 0.5 & & 1 & 1.6 & 1.0 & 0.9 & 1.0 \\
\hline & 10 & 14.0 & 10.4 & 9.3 & 9.9 & & 10 & 12.2 & 10.2 & 9.8 & 10.1 \\
20 & 5 & 7.9 & 5.2 & 4.4 & 4.9 & 40 & 5 & 6.4 & 5.1 & 4.9 & 5.1 \\
& 2.5 & 4.2 & 2.4 & 2.1 & 2.4 & & 2.5 & 3.4 & 2.7 & 2.4 & 2.6 \\
& 1 & 1.9 & 1.0 & 0.6 & 0.8 & & 1 & 1.5 & 1.1 & 0.9 & 1.0 \\
\hline
\end{tabular}


Tabela 4.5: Taxas de rejeição para o teste da hipótese $H^{\prime}{ }_{2}: \gamma_{1}=0$.

Valores da variável independente obtidos da $\mathrm{N}(0,1)$.

\begin{tabular}{rrrrrrrrrrrr}
\hline$n$ & $\alpha(\%)$ & $L R$ & $L R^{*}$ & $S_{R}$ & $S_{R}^{*}$ & $n$ & $\alpha(\%)$ & $L R$ & $L R^{*}$ & $S_{R}$ & $S_{R}^{*}$ \\
\hline & 10 & 20.7 & 14.3 & 4.7 & 7.7 & & 10 & 12.9 & 10.6 & 8.5 & 9.8 \\
10 & 5 & 13.6 & 8.4 & 1.7 & 3.3 & 30 & 5 & 6.9 & 5.3 & 3.9 & 4.8 \\
& 2.5 & 9.1 & 4.9 & 0.6 & 1.4 & & 2.5 & 3.5 & 2.5 & 1.9 & 2.3 \\
& 1 & 5.3 & 2.5 & 0.1 & 0.4 & & 1 & 1.4 & 1.0 & 0.7 & 0.9 \\
\hline \multirow{2}{*}{20} & 10 & 13.8 & 10.6 & 7.7 & 9.4 & & 10 & 11.5 & 10.0 & 8.8 & 9.9 \\
& 5 & 7.8 & 5.6 & 3.7 & 4.8 & 40 & 5 & 6.6 & 5.5 & 4.8 & 5.3 \\
& 2.5 & 4.4 & 2.9 & 1.7 & 2.3 & & 2.5 & 3.5 & 2.9 & 2.4 & 2.8 \\
& 1 & 2.1 & 1.3 & 0.6 & 0.8 & & 1 & 1.6 & 1.2 & 0.7 & 0.9 \\
\hline
\end{tabular}

Tabela 4.6: Taxas de rejeição para o teste da hipótese $H_{3}^{\prime}: \beta_{1}=0$.

Valores da variável independente obtidos da $\mathrm{N}(0,1)$.

\begin{tabular}{rrrrrrrrrrrr}
\hline$n$ & $\alpha(\%)$ & $L R$ & $L R^{*}$ & $S_{R}$ & $S_{R}^{*}$ & $n$ & $\alpha(\%)$ & $L R$ & $L R^{*}$ & $S_{R}$ & $S_{R}^{*}$ \\
\hline & 10 & 20.0 & 13.9 & 6.2 & 6.6 & & 10 & 12.5 & 10.3 & 9.8 & 9.9 \\
10 & 5 & 14.0 & 9.5 & 2.9 & 3.0 & 30 & 5 & 6.8 & 5.3 & 4.6 & 4.9 \\
& 2.5 & 10.5 & 7.3 & 1.8 & 2.0 & & 2.5 & 3.7 & 2.7 & 2.1 & 2.4 \\
& 1 & 7.9 & 5.8 & 1.4 & 1.2 & & 1 & 1.7 & 1.2 & 0.8 & 1.1 \\
\hline \multirow{2}{*}{20} & 10 & 13.3 & 10.1 & 9.3 & 9.3 & & 10 & 11.6 & 10.0 & 9.8 & 9.8 \\
& 5 & 7.5 & 5.6 & 4.3 & 4.6 & 40 & 5 & 6.0 & 4.9 & 4.7 & 4.9 \\
& 2.5 & 4.8 & 3.4 & 2.2 & 2.6 & & 2.5 & 3.0 & 2.3 & 2.1 & 2.3 \\
& 1 & 2.8 & 2.1 & 1.5 & 1.3 & & 1 & 1.4 & 1.1 & 0.8 & 1.0 \\
\hline
\end{tabular}


Tabela 4.7: Taxas de rejeição para o teste da hipótese $H_{1}^{\prime}: \beta_{1}=\gamma_{1}=0$.

Valores da variável independente obtidos da $\operatorname{LN}\left(0,1.25^{2}\right)$.

\begin{tabular}{rrrrrrrrrrrr}
\hline$n$ & $\alpha(\%)$ & $L R$ & $L R^{*}$ & $S_{R}$ & $S_{R}^{*}$ & $n$ & $\alpha(\%)$ & $L R$ & $L R^{*}$ & $S_{R}$ & $S_{R}^{*}$ \\
\hline & 10 & 17.2 & 8.4 & 9.0 & 10.9 & & 10 & 16.5 & 11.3 & 8.7 & 9.6 \\
10 & 5 & 11.8 & 4.8 & 6.1 & 7.1 & 30 & 5 & 9.2 & 5.8 & 5.3 & 5.0 \\
& 2.5 & 8.3 & 2.6 & 4.2 & 3.1 & & 2.5 & 5.6 & 3.3 & 3.7 & 0.0 \\
& 1 & 5.2 & 1.5 & 2.6 & 0.0 & & 1 & 2.9 & 1.5 & 2.1 & 0.0 \\
\hline \multirow{3}{*}{20} & 10 & 20.0 & 12.8 & 8.5 & 9.4 & & 10 & 14.7 & 11.1 & 8.7 & 9.8 \\
& 5 & 12.7 & 6.9 & 5.6 & 5.1 & 40 & 5 & 8.5 & 5.9 & 5.3 & 4.9 \\
& 2.5 & 8.0 & 3.8 & 3.8 & 0.0 & & 2.5 & 4.8 & 2.9 & 3.5 & 0.0 \\
& 1 & 4.3 & 1.6 & 2.3 & 0.0 & & 1 & 2.1 & 1.1 & 2.0 & 0.0 \\
\hline
\end{tabular}

Tabela 4.8: Taxas de rejeição para o teste da hipótese $H_{2}^{\prime}: \gamma_{1}=0$.

Valores da variável independente obtidos da $\operatorname{LN}\left(0,1.25^{2}\right)$.

\begin{tabular}{rrrrrrrrrrrr}
\hline$n$ & $\alpha(\%)$ & $L R$ & $L R^{*}$ & $S_{R}$ & $S_{R}^{*}$ & $n$ & $\alpha(\%)$ & $L R$ & $L R^{*}$ & $S_{R}$ & $S_{R}^{*}$ \\
\hline \multirow{4}{*}{10} & 10 & 18.2 & 8.6 & 0.0 & 0.2 & & 10 & 16.5 & 11.1 & 4.6 & 7.5 \\
& 5 & 12.6 & 4.9 & 0.0 & 0.0 & 30 & 5 & 9.9 & 6.0 & 2.0 & 3.3 \\
& 2.5 & 9.2 & 2.6 & 0.0 & 0.0 & & 2.5 & 6.0 & 3.3 & 1.3 & 1.7 \\
& 1 & 6.0 & 0.9 & 0.0 & 0.0 & & 1 & 3.3 & 1.3 & 0.8 & 0.9 \\
\hline & 10 & 21.4 & 13.2 & 3.2 & 5.7 & & 10 & 15.3 & 11.1 & 6.1 & 8.7 \\
20 & 5 & 14.0 & 7.0 & 1.9 & 2.9 & 40 & 5 & 8.8 & 5.7 & 2.8 & 4.3 \\
& 2.5 & 8.6 & 3.8 & 1.2 & 2.1 & & 2.5 & 5.0 & 2.8 & 1.8 & 2.4 \\
& 1 & 5.0 & 1.7 & 0.7 & 1.2 & & 1 & 2.3 & 1.2 & 0.9 & 1.2 \\
\hline
\end{tabular}


Tabela 4.9: Taxas de rejeição para o teste da hipótese $H_{3}^{\prime}: \beta_{1}=0$.

Valores da variável independente obtidos da $\operatorname{LN}\left(0,1.25^{2}\right)$.

\begin{tabular}{rrrrrrrrrrrr}
\hline$n$ & $\alpha(\%)$ & $L R$ & $L R^{*}$ & $S_{R}$ & $S_{R}^{*}$ & $n$ & $\alpha(\%)$ & $L R$ & $L R^{*}$ & $S_{R}$ & $S_{R}^{*}$ \\
\hline & 10 & 19.7 & 10.0 & 17.2 & 13.9 & & 10 & 16.9 & 11.6 & 9.0 & 9.7 \\
10 & 5 & 14.8 & 6.6 & 11.0 & 9.9 & 30 & 5 & 10.1 & 6.4 & 3.6 & 4.1 \\
& 2.5 & 11.6 & 4.7 & 7.3 & 7.7 & & 2.5 & 6.2 & 3.4 & 1.0 & 1.6 \\
& 1 & 8.6 & 3.5 & 4.6 & 6.5 & & 1 & 3.2 & 1.5 & 0.3 & 0.6 \\
\hline \multirow{2}{*}{20} & 10 & 22.5 & 15.1 & 7.7 & 8.9 & & 10 & 15.0 & 11.1 & 9.6 & 10.0 \\
& 5 & 15.1 & 9.0 & 3.2 & 3.4 & 40 & 5 & 8.7 & 5.7 & 3.8 & 4.2 \\
& 2.5 & 10.3 & 5.5 & 1.6 & 2.0 & & 2.5 & 4.8 & 2.9 & 1.1 & 1.9 \\
& 1 & 6.3 & 3.4 & 1.2 & 1.1 & & 1 & 2.3 & 1.2 & 0.2 & 0.6 \\
\hline
\end{tabular}




\subsection{Uma aplicação a dados reais}

Os dados da Tabela 4.10, obtidos de Simonoff e Tsai (1994), representam as taxas mensais de retorno de ações de mercado $(\mathrm{X})$ e da Corporação Acme Cleveland (Y), no período de janeiro de 1986 a dezembro de 1990. Estes autores sugerem que os dados sejam modelados através do modelo normal heterocedástico dado em (1.1) considerando as seguintes componentes sistemáticas para a média e o parâmetro de precisão

$$
\mu_{l}=\beta_{2}+\beta_{1} x_{l}
$$

e

$$
-\log \left(\phi_{l}\right)=\gamma_{2}+\gamma_{i 1} x_{l}
$$

para $l=1, \ldots, 59$ (o caso referente a outubro de 1987 foi eliminado por ter sido considerado um ponto discrepante no conjunto de valores). Nosso objetivo é testar $H_{0}: \gamma_{1}=0$ contra $H: \gamma_{1} \neq 0$, ou seja, se o modelo é homocedástico. Através do procedimento de estimação proposto na Seção 1.2, encontramos, sob o modelo irrestrito, as seguintes estimativas de máxima verossimilhança, $\hat{\beta}_{2}=-0,00492, \hat{\beta}_{1}=1.25270, \hat{\gamma}_{2}=-4,41000$ e $\hat{\gamma}_{1}=8,09200$. Sob $H_{0}$, obtivemos $\tilde{\beta}_{2}=-0,00951, \tilde{\beta}_{1}=1,17240$ e $\tilde{\gamma}_{2}=-4,73910$. As estatísticas da razão de verossimilhança e escore vêm das expressões apresentadas nas Seções 2.3.1 e 3.3.1, respectivamente, fornecendo os valores $L R=3,33$ e $S_{R}=2$.69. Os níveis descritivos, obtidos da distribuição $\chi_{1}^{2}$ são iguais a $6,8 \%$, para $L R$ e $10,1 \%$, para $S_{R}$. Para obter as estatísticas corrigidas utilizamos, respectivamente, os resultados das Seções 2.3.1 e 3.3.1. Encontramos $L R^{*}=3,12$ e $S_{R}^{*}=2,89$. Os níveis descritivos são dados agora por $7,73 \%$ e $8,91 \%$, respectivamente. Como esperado, observamos que a diferença entre os níveis descritivos das estatísticas corrigidas é menor do que a diferença entre os níveis descritivos das estatísticas não corrigidas. Além disto, através dos níveis descritivos das estatísticas corrigidas concluímos que há evidências, ao nível de $10 \%$ de significância, de que o modelo adotado para estes dados é heterocedástico. 
Tabela 4.10: Taxas mensais de retorno de ações de mercado (X) e da Corporação Acme Cleveland (Y), no período de janeiro de 1986 a dezembro de 1990.

\begin{tabular}{|c|c|c|c|c|c|}
\hline Month & Marketreturn & Acmereturn & Month & Marketreturn & Acmereturn \\
\hline Janeiro 1986 & $-0,061134$ & 0,030160 & Julho 1988 & $-0,061718$ & $-0,110515$ \\
\hline Fevereiro 1986 & 0,008220 & $-0,165457$ & Agosto 1988 & $-0,101710$ & $-0,168769$ \\
\hline Março 1986 & $-0,007381$ & 0,080137 & Setembro 1988 & $-0,032705$ & $-0,135585$ \\
\hline Abril 1986 & $-0,067561$ & $-0,109917$ & Outubro 1988 & $-0,045334$ & $-0,084077$ \\
\hline Maio 1986 & $-0,006238$ & $-0,114853$ & Novembro 1988 & $-0,079288$ & $-0,164550$ \\
\hline Junho 1986 & $-0,044251$ & $-0,099254$ & Dezembro 1988 & $-0,036233$ & 0,150269 \\
\hline Julho 1986 & $-0,112070$ & $-0,226846$ & Janeiro 1989 & $-0,011494$ & $-0,015672$ \\
\hline Agosto 1986 & 0,030226 & 0,073445 & Fevereiro 1989 & $-0,093729$ & $-0,037860$ \\
\hline Setembro 1986 & $-0,129556$ & $-0,143064$ & Março 1989 & $-0,065215$ & $-0,074712$ \\
\hline Outubro 1986 & 0,001349 & 0,034776 & Abril 1989 & $-0,037113$ & $-0,108530$ \\
\hline Novembro 1986 & $-0,033679$ & $-0,063375$ & Maio 1989 & $-0,044399$ & $-0,036769$ \\
\hline Dezembro 1986 & $-0,072795$ & $-0,058735$ & Junho 1989 & $-0,084412$ & 0,023912 \\
\hline Janeiro 1987 & 0,073396 & 0,050214 & Julho 1989 & 0,003444 & $-0,078430$ \\
\hline Fevereiro 1987 & $-0,011618$ & 0,111165 & Agosto 1989 & $-0,056760$ & $-0,132199$ \\
\hline Março 1987 & $-0,026852$ & $-0,127492$ & Setembro 1989 & $-0,078970$ & $-0,110141$ \\
\hline Abril 1987 & $-0,040356$ & 0,054522 & Outubro 1989 & $-0,105367$ & $-0,126302$ \\
\hline Maio 1987 & $-0,047539$ & $-0,072918$ & Novembro 1989 & $-0,038634$ & $-0,095730$ \\
\hline Junho 1987 & $-0,001732$ & $-0,058979$ & Dezembro 1989 & $-0,043261$ & 0,065740 \\
\hline Julho 1987 & $-0,008899$ & 0,236147 & Janeiro 1990 & $-0,139773$ & $-0,120056$ \\
\hline Agosto 1987 & $-0,020837$ & $-0,094778$ & Fevereiro 1990 & $-0,059094$ & $-0,085205$ \\
\hline Setembro 1987 & $-0,084811$ & $-0,135669$ & Março 1990 & $-0,057736$ & $-0,130433$ \\
\hline Outubro 1987 & $-0,262077$ & $-0,284796$ & Abril 1990 & $-0,102524$ & $-0,116728$ \\
\hline Novembro 1987 & $-0,110167$ & $-0,171494$ & Maio 1990 & 0,023881 & $-0,078039$ \\
\hline Dezembro 1987 & 0,034955 & 0,242616 & Junho 1990 & $-0,079116$ & $-0,170322$ \\
\hline Janeiro 1988 & 0,012688 & $-0,063518$ & Julho 1990 & $-0,078965$ & $-0,077727$ \\
\hline Fevereiro 1988 & $-0,002170$ & $-0,117677$ & Agosto 1990 & $-0,161359$ & $-0,277035$ \\
\hline Março 1988 & $-0,073462$ & 0,201674 & Setembro 1990 & $-0,119376$ & $-0,207595$ \\
\hline Abril 1988 & $-0,043419$ & $-0,147728$ & Outubro 1990 & $-0,076008$ & $-0,070515$ \\
\hline Maio 1988 & $-0,054730$ & $-0,170885$ & Novembro 1990 & $-0,006444$ & $-0,046274$ \\
\hline Junho 1988 & $-0,011755$ & $-0,014893$ & Dezembro 1990 & $-0,026401$ & $-0,190834$ \\
\hline
\end{tabular}




\section{Conclusão}

Neste trabalho obtivemos correções tipo-Bartlett para estatísticas escore de testes sobre a média e/ou o parâmetro de precisão em modelos normais heterocedásticos. Baseados nos resultados de Botter e Cordeiro (1997) e de Cordeiro (1993), apresentamos correções de Bartlett para as estatísticas da razão de verossimilhança destes mesmos testes. Através de resultados de estudos de simulações verificamos que as estatísticas corrigidas têm tamanhos empíricos mais próximos dos nominais do que as estatísticas não corrigidas, em amostras finitas. Estes resultados mostraram, também, que a estatística escore não corrigida tem tamanho empírico mais próximo do nominal do que a estatística da razão de verossimilhança sem correção. Assim sendo, em testes de hipóteses no modelo normal heterocedástico, é natural a escolha da estatística escore por requerer somente estimação sob a hipótese nula. Como sugestões para pesquisas futuras, consideramos interessante o estudo da correção do ponto crítico de estatísticas escore, assim como a obtenção de estatísticas escore monótonas em modelos normais heterocedásticos. 


\section{Apêndice}

Neste apêndice, apresentamos os cumulantes de derivadas do logaritmo da função de verossimilhança total no modelo normal heterocedástico definido em (1.1) e as expressões gerais para as quantidades $A_{1}, A_{2}$ e $A_{3}$ necessárias para a obtenção das correções tipo-Bartlett para a estatística $S_{R}$.

Utilizamos notações com índices de letras minúsculas para as derivadas em relação a $\beta$ e as notações com índices de letras maiúsculas, para as derivadas em relação a $\gamma$. Assim, temos $U_{r}=\partial \ell / \partial \beta_{r}, U_{R}=\partial \ell / \partial \gamma_{R}, U_{r S}=\partial^{2} \ell / \partial \beta_{r} \ell \gamma_{S}, U_{r S T}=\partial^{3} \ell / \partial \beta_{r} \partial \gamma_{S} \partial \gamma_{T}, U_{r S T U}=$ $\partial^{4} \ell / \partial \beta_{r} \partial \gamma_{S} \partial \gamma_{T} \partial \gamma_{U}$. Para os cumulantes dessas derivadas, definimos as notações $\kappa_{r S}=$ $E\left(U_{r S}\right), \kappa_{r S T}=E\left(U_{r S T}\right), \kappa_{r, s}=E\left(U_{r} U_{s}\right), \kappa_{r, S T}=E\left(U_{r} U_{S T}\right), \kappa_{r s t u}=E\left(U_{r s t u}\right), \kappa_{R S, t u}=$ $E\left(U_{R S} U_{t u}\right)-\kappa_{R S} \kappa_{t u}$, etc. Logo,

$$
\begin{gathered}
\kappa_{r s}=-\sum_{l=1}^{n}(\phi w)_{l} x_{l r} x_{l s}, \\
\kappa_{R S}=-2^{-1} \sum_{l=1}^{n}\left(\phi^{-2} \phi_{1}^{2}\right)_{l} s_{l R} s_{l S}, \\
\kappa_{r s t}=-3 \sum(\phi f)_{l} x_{l r} x_{l s} x_{l t}, \\
\kappa_{r s T}=-\sum\left(\phi_{1} w\right)_{l} x_{l r} x_{l s} s_{l T}, \\
\kappa_{R S T}=2^{-1} \sum \phi_{l}^{-} 2\left(2 \phi^{-} 1 \phi_{1}^{3}-3 \phi_{1} \phi_{2}\right)_{l} s_{l R} s_{l S} s_{l T}, \\
\kappa_{r, s t}=\sum(\phi f)_{l} x_{l r} x_{l s} x_{l t}, \\
\kappa_{r, s T}=\sum\left(\phi_{1} w\right)_{l} x_{l r} x_{l s} s_{l T}, \\
\kappa_{r S}=\kappa_{r S T}=\kappa_{r, S T}=\kappa_{R, s t}=\kappa_{R, s T}=0, \\
\kappa_{R, S T}=2^{-1} \sum\left(\phi^{-} 2 \phi_{1}^{2} \phi_{2}\right)_{l} s_{l R} s_{l S} s_{l T}, \\
\kappa_{r, s, T}=-\sum\left(\phi_{1} w\right)_{l} x_{l r} x_{l s} s_{l T}, \\
\kappa_{R, S, T}=-\sum\left(\phi^{-} 3 \phi_{1}^{3}\right)_{l} s_{l R} s_{l S} s_{l T}, \\
\kappa_{r, S, T}=\kappa_{r, s, t}=\kappa_{r, s, t, u}=\kappa_{r, s, t, U}=\kappa_{r, S, T, U}=0, \\
\kappa_{r, s, T, U}=2 \sum\left(\phi^{-} 1 \phi_{1}^{2} w\right)_{l} x_{l r} s_{l s} s_{l T} s_{l U}, \\
\kappa_{R, S, T, U}=3 \sum\left(\phi^{-} 4 \phi_{1}^{4}\right)_{l} s_{l R} s_{l S} s_{l T} s_{l U} . \\
\kappa_{r, s, t u}=\kappa_{R, s, t U}=\kappa_{R, s, T, U}=\kappa_{R, S, t u}=\kappa_{R, S, t U}=0,
\end{gathered}
$$




$$
\begin{aligned}
\kappa_{r, s, T U} & =-\sum\left(\phi_{2} w\right)_{l} x_{l r} x_{l s} s_{l T} s_{l U}, \\
\kappa_{R, s, t u} & =-\sum\left(\phi_{1} f\right)_{l} s_{l R} x_{l s} x_{l t} x_{l u}, \\
\kappa_{R, S, T U} & =-\sum\left(\phi^{-} 3 \phi_{1}^{2} \phi_{2}\right)_{l} s_{l R} s_{l S} s_{l T} s_{l U}, \\
\kappa_{r s T U} & =-\sum\left(\phi_{2} w\right)_{l} x_{l r} x_{l s} s_{l T} s_{l U}, \\
\kappa_{R S T U} & =-\sum\left[2^{-1} \phi^{-2}\left(6 \phi^{-2} \phi_{1}^{4} 12 \phi^{-1} \phi_{1}^{2} \phi_{2}+3 \phi_{2}^{2}+4 \phi_{1} \phi_{3}\right)\right]_{l} x_{l R} x_{l S} s_{l T} s_{l U}, \\
\kappa_{r s t u} & =-\sum(a \phi)_{l} x_{l r} x_{l s} x_{l t} x_{l u},
\end{aligned}
$$

onde $a=3\left(\frac{d^{2} \mu}{d \eta^{2}}\right)^{2}+4 \frac{d^{3} \mu}{d \eta^{3}}$

As quantidades $A_{1},\left(A_{2}\right)$ e $\left(A_{3}\right)$ são dadas por

$$
\begin{aligned}
& A_{1}=3 \sum_{\beta, \gamma}\left(\kappa_{i_{1} i_{2} i_{3}}+2 \kappa_{i_{1}, i_{2} i_{3}}\right)\left(\kappa_{i_{4} i_{5} i_{6}}+2 \kappa_{i_{4} i_{5}, i_{6}}\right) a_{i_{1} i_{2}} a_{i_{5} i_{6}} m_{i_{3} i_{4}} \\
& -6 \sum_{\beta, \gamma}\left(\kappa_{i_{1} i_{2} i_{3}}+2 \kappa_{i_{1}, i_{2} i_{3}}\right) \kappa_{i_{4}, i_{5}, i_{6}} a_{i_{1} i_{2}} a_{i_{3} i_{4}} m_{i_{5} i_{6}} \\
& +6 \sum_{\beta, \gamma}\left(\kappa_{i_{1}, i_{2} i_{3}}-\kappa_{i_{1}, i_{2}, i_{3}}\right)\left(\kappa_{i_{4} i_{5} i_{6}}+2 \kappa_{i_{4} i_{5}, i_{6}}\right) a_{i_{2} i_{5}} a_{i_{3} i_{6}} m_{i_{1} i_{4}} \\
& -6 \sum_{\beta, \gamma}\left(\kappa_{i_{1}, i_{2}, i_{3}, i_{4}}+\kappa_{i_{1}, i_{2}, i_{3} i_{4}}\right) a_{i_{3} i_{4}} m_{i_{1} i_{2}}, \\
& A_{2}={ }^{\prime}-3 \sum_{\beta, \gamma} \kappa_{i_{1}, i_{2}, i_{3}} \kappa i_{4}, i_{5}, i_{6} a_{i_{3} i_{4}} m_{i_{1} i_{2}} m_{i_{5} i_{6}} \\
& 1+6 \sum_{\beta, \gamma}\left(\kappa_{i_{1} i_{2} i_{3}}+2 \kappa_{i_{1}, i_{2} i_{3}}\right) \kappa_{i 4, i_{5}, i_{6}} a_{i_{1} i_{2}} m_{i_{3} i_{4}} m_{i_{5} i_{6}} \\
& \supseteq-6 \sum_{\beta, \gamma} \kappa_{i_{1}, i_{2}, i_{3}} \kappa_{i_{4}, i_{5}, i_{6}} a_{i_{3} i_{6}} m_{i_{1} i_{4}} m_{i_{2} i_{5}} \\
& +3 \sum_{\beta, \gamma} \kappa_{i_{1}, i_{2}, i_{3} i_{4}} m_{i_{1} i_{2}} m_{i_{3} i_{4}} \text {, } \\
& A_{3}=3 \sum_{\beta, \gamma} \kappa_{i_{1}, i_{2}, i_{3}} \kappa_{i_{4}, i_{5}, i_{6}} m_{i_{1} i_{2}} m_{i_{3} i_{4}} m_{i_{5} i_{6}} \\
& +2 \sum_{\beta, \gamma} \kappa_{i_{1}, i_{2}, i_{3}} \kappa_{i_{4}, i_{5}, i_{6}} m_{i_{1} i_{4}} m_{i_{2} i_{5}} m_{i_{3} i_{6}},
\end{aligned}
$$

onde os índices $i_{1}, \ldots, i_{6}$ variam sobre todos os componentes dos vetores $\beta$ e $\gamma$ e $\sum_{\beta, \gamma}$ denota o somatório sobre todas as combinações dos $p+q$ parâmetros $\beta_{1}, \ldots, \beta_{p}, \gamma_{1}, \ldots, \gamma_{q}$. 


\section{Referências Bibliográficas}

ATTFIELD, C.L.F. (1991). A Bartlett-adjustment factor to the likelihood ratio test for homoskedasticity in the linear model. Economics Letters 37, 119-123.

AUBIN E.C.Q. e CORDEIRO, G.M.(1998). Some adjusted likelihood ratio tests for heteroscedasticit regression models. Brazilian Journal of Probability and Statistics, 2, 131-148

BARTLETT, M.S. (1937). Properties of sufficiency and statistical tests. Procedings of the Royal Society of London A. 160, 268-282.

BOTTER, D.A. e CORDEIRO, G.M. (1997). Bartlett corrections for generalized linear models with dispersion covariates. Communications in Statistics-Theory and Methods 26.

BREUSCH, T.S. (1979). Conflict among criteria for testing hypotheses: Extensions and comments. Econometrica 47, 203-207.

BREUSCH, T. S. e PAGAN, A.R. (1979). A simple test for heteroskedasticity end random coefficient variation. Econometrica 47, 1287-1294.

CHANDRA, T.K. (1985). Asymptotic expansions of perturbed chi-square variables. Sankhyā A 47, 100-110.

CHANDRA, T.K. e GHOSH, J.K. (1979). Valid asymptotic expansions of the likelihood ratio statistic and other perturbed chi-square variables. Sankhyā $A$ 41, 22-47.

CHAR, B.W., GEDDES, K.O., GONNET, G.R., MONAGAN, M.B. e WATT, S.M. (1988). MAPLE Reference Manual. Waterloo, Watcom Publications Ltd. 95p.

CHESHER, A., SMITH, R. (1995). Bartlett corrections to likelihood ratio tests. biomética, 82, 433-436. 
COOK, R.D. e WEISBERG. S. (1983). Diagnostics for heteroskedasticity in regression. Biometrica 70, 1-10.

CORDEIRO, G.M. (1987). On the corrections to the likelihood ratio statistics. Biometrica 74, 265-274.

CORDEIRO, G.M. (1993a). Bartlett correction and bias correction for two heteroscedastics regression models. Communications in Statistics-Theory and Methods 22, 169-188.

CORDEIRO, G.M. (1993b). General matrix formulae for computing Bartlett corrections. Statistics and Probability Letters 16, 11-18.

CORDEIRO G.M. e FERRARI, S.L.P. (1991). A general method for approximating to the distribuition of some statistics. Biometrika 78, 573-582.

CORDEIRO, G.M., FERRARI, S.L.P. e CYSNEIROS, A.H.M.A. (1998). A formula to improve score test statistics. Journal of Statistical Computation and Simulation 62, 123136.

CRIBARI-NETO, F. e FERRARI, S. L.P. (1995a). An improved Lagrange multiplier test for heteroskedasticity. Communications in Statistics-Computation and Simulation 24, 31-44.

CRIBARI-NETO, F. e FERRARI, S. L.P. (1995b). Bartlett- corrected tests for heteroskedastic linear models. Economics Letters 48, 113-118.

CRIBARI-NETO, F. e ZARKOS, S.G. (1999). Bootstrap methods for heteroskedastic regression models: evidence on estimation and testing. Econometric Reviews 18, 211-228.

COX, D.R. e HINKLEY, D.V. (1974). Theoretical Statistics. New York: John Wiley. 
COX, D.R. e REID, N. (1987). Parameter orthogonality and approximate conditional inference (with discussion). Journal of the Royal Statistical Society B 49, 1-39.

EVANS, G.B.A e SAVIN, N.E. (1982). Conflict among and criteria revisited; the W, LR and LM tests. Econometrica 50, 737-748.

GODFREY, L.G. (1978). Testing for multiplicative heteroskedasticity. Journal of Econometrics 8, 227-236.

H.ARRIS, P. (1985). An asymptotic expansion for the null distribution of the efficient score statistic. Biometrika 72, 653-659.

HAYAKAWA, T. (1977). The likelihood ratio criterion and the asymtoptic expansion of its distribution. Annals of the Institute of Statistical Mathematics A 29, 359-78, e Corrigenda in Annals of the Institute of Statistical Mathematics 39, 681 (1987).

HOLLAS, E.A. (1991). Performance of Bartlett adjustment for certain likelihood ratio tests. Communications in Statistics B 20, 449-462.

HONDA, Y. (1988). A size correction to the lagrange multiplier test for heteroskedasticity. Journal of Econometrics 38, 375-386.

JENSEN, J.L. (1993). A historical sketch and some new results on the improved log likelihood ratio statistic. Scandinavian Journal of Statistical 20, 1-15.

L.AWLEY, D. N. (1956). A general method for approximating to the distribuition of the likelihood ratio criteria. Biometrika 43, 295-303.

NELDER, J.A. e WEDDERBURN, R.W.M. (1972). Generalized linear models. Journal of the Royal Statistical Society 135, 370-384. 
R.AO C.R. (1948). Large sample tests of statistical hypotheses concerning several parameters with applications to problems of estimation. Proceedings of the Cambridge Philosophical Society 44, 50-57.

SIMONOFF, S.J. e TSAI, CHIH-LING (1994). Use of modified profile likelihood for improved tests of constancy of variance in regression. Applied Statistics 43, 357-370,

S.IYTH, G.K. (1989). Generalized linear models with varyng dispersion. Journal of the Royal Statistical Society B 51, 47-60.

WOLFRAM, S. (1991). Mathematica a System for Doing Mathematics by Computer. 2. ed. New York: Addison-Wesley. 